\title{
Anisotropic and plane-selective migration of the carbon vacancy in SiC: Theory and experiment
}

\author{
M. E. Bathen,,${ }^{1,}$ J. Coutinho, ${ }^{2}$ H. M. Ayedh,,${ }^{1}$ J. Ul Hassan, ${ }^{3}$ I. Farkas, ${ }^{3}$ S. Öberg, ${ }^{4}$ Y. K. Frodason, ${ }^{1}$ \\ B. G. Svensson, ${ }^{1}$ and L. Vines ${ }^{1}$ \\ ${ }^{1}$ Department of Physics/Centre for Materials Science and Nanotechnology, University of Oslo, N-0316 Oslo, Norway \\ ${ }^{2}$ Department of Physics and I3N, University of Aveiro, Campus Santiago, 3810-193 Aveiro, Portugal \\ ${ }^{3}$ Department of Physics, Chemistry and Biology, Linköping University, SE-58183 Linköping, Sweden \\ ${ }^{4}$ Department of Engineering Sciences and Mathematics, Luleå University of Technology, SE-97187 Luleå, Sweden
}

(Received 25 February 2019; revised manuscript received 3 May 2019; published 8 July 2019)

\begin{abstract}
We investigate the migration mechanism of the carbon vacancy $\left(V_{\mathrm{C}}\right)$ in silicon carbide (SiC) using a combination of theoretical and experimental methodologies. The $V_{\mathrm{C}}$, commonly present even in state-of-the-art epitaxial $\mathrm{SiC}$ material, is known to be a carrier lifetime killer and therefore strongly detrimental to device performance. The desire for $V_{\mathrm{C}}$ removal has prompted extensive investigations involving its stability and reactivity. Despite suggestions from theory that $V_{\mathrm{C}}$ migrates exclusively on the $\mathrm{C}$ sublattice via vacancy-atom exchange, experimental support for such a picture is still unavailable. Moreover, the existence of two inequivalent locations for the vacancy in $4 \mathrm{H}-\mathrm{SiC}$ [hexagonal, $V_{\mathrm{C}}(h)$, and pseudocubic, $V_{\mathrm{C}}(k)$ ] and their consequences for $V_{\mathrm{C}}$ migration have not been considered so far. The first part of the paper presents a theoretical study of $V_{\mathrm{C}}$ migration in $3 \mathrm{C}$ - and $4 \mathrm{H}-\mathrm{SiC}$. We employ a combination of nudged elastic band (NEB) and dimer methods to identify the migration mechanisms, transition state geometries, and respective energy barriers for $V_{\mathrm{C}}$ migration. In $3 \mathrm{C}$-SiC, $V_{\mathrm{C}}$ is found to migrate with an activation energy of $E_{\mathrm{A}}=4.0 \mathrm{eV}$. In $4 \mathrm{H}-\mathrm{SiC}$, on the other hand, we anticipate that $V_{\mathrm{C}}$ migration is both anisotropic and basal-plane selective. The consequence of these effects is a slower diffusivity along the axial direction, with a predicted activation energy of $E_{\mathrm{A}}=4.2 \mathrm{eV}$, and a striking preference for basal migration within the $h$ plane with a barrier of $E_{\mathrm{A}}=3.7 \mathrm{eV}$, to the detriment of the $k$-basal plane. Both effects are rationalized in terms of coordination and bond angle changes near the transition state. In the second part, we provide experimental data that corroborates the above theoretical picture. Anisotropic migration of $V_{\mathrm{C}}$ in $4 \mathrm{H}-\mathrm{SiC}$ is demonstrated by deep level transient spectroscopy (DLTS) depth profiling of the $\mathrm{Z}_{1 / 2}$ electron trap in annealed samples that were subject to ion implantation. Activation energies of $E_{\mathrm{A}}=(4.4 \pm 0.3) \mathrm{eV}$ and $E_{\mathrm{A}}=(3.6 \pm 0.3) \mathrm{eV}$ were found for $V_{\mathrm{C}}$ migration along the $c$ and $a$ directions, respectively, in excellent agreement with the analogous theoretical values. The corresponding prefactors of $D_{0}=0.54 \mathrm{~cm}^{2} / \mathrm{s}$ and $0.017 \mathrm{~cm}^{2} / \mathrm{s}$ are in line with a simple jump process, as expected for a primary vacancy point defect.
\end{abstract}

DOI: 10.1103/PhysRevB.100.014103

\section{INTRODUCTION}

The properties of silicon carbide ( $\mathrm{SiC}$ ), including a wide band gap, large breakdown field, and radiation hardness, are highly advantageous for conceiving novel quantum, optical, and electronic devices [1,2], such as power MOSFETs [3] and nuclear detectors operating under harsh conditions [4]. Recent breakthroughs have established $\mathrm{SiC}$ as a leading candidate host for solid-state single-photon emitters (SPEs) and spin centers that are highly desirable for quantum computing devices. Behind these findings are the silicon vacancy $\left(V_{\mathrm{Si}}\right)$ in $4 \mathrm{H}-\mathrm{SiC}$ [5], as well as the nitrogen-vacancy $\left(\mathrm{N}_{\mathrm{C}} V_{\mathrm{Si}}\right)[5,6]$ and divacancy $\left(V_{\mathrm{C}} V_{\mathrm{Si}}\right)$ [6-8] centers in both $3 \mathrm{C}$ and $4 \mathrm{H}$ materials. On the other hand, for devices that essentially rely on the lifetime of charge carriers, defects remain a perennial threat. Unlike extended defects which have been largely eliminated from state-of-the-art epitaxial material, point defects and impurities still limit the minority carrier lifetime in $\mathrm{SiC}$ by acting as carrier traps and recombination centers.

*m.e.bathen@fys.uio.no
The carbon vacancy $\left(V_{\mathrm{C}}\right)$, in particular, is a prominent trap with strong and detrimental impact on the carrier lifetime of 4H-SiC [9-11]. Being omnipresent even in state-of-the-art epitaxial material [12] (where $V_{\mathrm{C}}$ is typically found in concentrations in the range $10^{12}-10^{13} \mathrm{~cm}^{-3}$ ), the $V_{\mathrm{C}}$ effectively limits the carrier lifetime to below $5 \sim \mu \mathrm{s}$, which is too low for devices operating with blocking voltages above $10 \mathrm{kV}$ [13]. Understanding the behavior of this defect, especially during common device processing steps such as ion implantation and high temperature heat treatments, is therefore crucial for realizing the full potential of $\mathrm{SiC}$.

In $4 \mathrm{H}-\mathrm{SiC}$, and disregarding any departure from the perfect vacancy structure due to pseudo-Jahn-Teller distortions [14], $V_{\mathrm{C}}$ may occur in two configurations, namely at pseudocubic and hexagonal sites $\left[V_{\mathrm{C}}(k)\right.$ and $\left.V_{\mathrm{C}}(h)\right]$, essentially differing by some of their second neighbors and more remote ligands. The $3 \mathrm{C}-\mathrm{SiC}$ cubic phase is isotropic, and only one $V_{\mathrm{C}}$ configuration can be found. Both $V_{\mathrm{C}}$ configurations in $4 \mathrm{H}-\mathrm{SiC}$ are negative- $U$ double acceptors, meaning that single-negative charge states are metastable and accessible only upon persistent illumination [15]. In n-type $4 \mathrm{H}-\mathrm{SiC}$, a twofold electron emission from $V_{\mathrm{C}}$ can be detected by deep level transient 
spectroscopy (DLTS), being manifested as a prominent peak at about $290 \mathrm{~K}$ (labeled $\mathrm{Z}_{1 / 2}$ ). This peak was connected to the superposition of $(-2 / 0)$ charge-state transitions of both $V_{\mathrm{C}}(k)$ and $V_{\mathrm{C}}(h)$ defects. Measurements of the activation energy for electron emission placed the transition at $0.67 \mathrm{eV}$ below the conduction band edge $\left(E_{\mathrm{c}}\right)$ [15]. As far as we are aware, no analogous DLTS peaks were detected in $3 \mathrm{C}-\mathrm{SiC}$. This could be explained if we consider that the valence band maxima of 3C- and 4H-SiC are essentially aligned (a small $\sim 60 \mathrm{meV}$ offset has been measured [16]), and that the LangerHeinrich rule applies to defects in different $\mathrm{SiC}$ polytypes [17], i.e., $V_{\mathrm{C}}$ levels are approximately pinned to the vacuum level regardless of the polytype [18]. With this in mind, we estimate the $(-2 / 0)$ transition of $V_{\mathrm{C}}$ in $3 \mathrm{C}-\mathrm{SiC}$ to be located at $\sim 0.3-0.4 \mathrm{eV}$ above $E_{\mathrm{c}}$.

First-principles calculations show that the charge-neutral $V_{\mathrm{C}}$ in $4 \mathrm{H}-\mathrm{SiC}$ has a low formation energy in the range $4.5-5 \mathrm{eV}[14,19]$, partly explaining its prevalence in as-grown (nonirradiated) material, apparently showing a high thermal stability [12,20,21]. It has been shown that reaching the thermodynamic equilibrium of the $V_{\mathrm{C}}$, at, e.g., $\sim 1500{ }^{\circ} \mathrm{C}$, requires less than $1 \mathrm{~h}$ and results in a $V_{\mathrm{C}}$ concentration of only $\sim 10^{11} \mathrm{~cm}^{-3}$ [13]. On the other hand, electron paramagnetic resonance (EPR) studies reported that $V_{\mathrm{C}}$ anneals out already at $1100{ }^{\circ} \mathrm{C}[22,23]$, with some traces remaining at $1600^{\circ} \mathrm{C}$. These observations could however be explained by a reaction with an impurity or defect that becomes mobile above $1100{ }^{\circ} \mathrm{C}$, and not necessarily by the disappearance of $V_{\mathrm{C}}$ upon its motion. Thus, despite indications that $V_{\mathrm{C}}$ could be mobile over a wide temperature window $\left(\sim 1100-1600{ }^{\circ} \mathrm{C}\right)$, both its diffusivity and underlying mechanism remain undetermined. At the above temperatures, intrinsic conditions apply to $\mathrm{SiC}$, and most likely $V_{\mathrm{C}}$ adopts the neutral charge state in both $3 \mathrm{C}$ and $4 \mathrm{H}-\mathrm{SiC}$. However, by noting that under these conditions the Fermi level is close to the donor transition of $V_{\mathrm{C}}$, we cannot entirely rule out a possible contribution of a smaller population of positively charged vacancies to the diffusivity.

Previous theoretical studies suggest that the migration mechanism of $V_{\mathrm{C}}$, in both $3 \mathrm{C}$ - and $4 \mathrm{H}-\mathrm{SiC}$, does not involve other defects. They indicate that $V_{\mathrm{C}}$ jumps are solely mediated by vacancy-atom exchange within the $\mathrm{C}$ sublattice [24,25]. For neutral $V_{\mathrm{C}}$ in $3 \mathrm{C}-\mathrm{SiC}$, the activation energy for migration via second-neighbor hopping was estimated at $E_{\mathrm{A}}=3.5 \mathrm{eV}$ using local density functional theory [24-26]. In order to mitigate the self-interaction error from the local functional, that figure was increased to $4.2 \mathrm{eV}$ after post correcting the total energies by means of ad-hoc shifts to the one-electron energies [26]. Other studies, using larger supercells and an efficient but less accurate self-consistent density-functionalbased tight-binding method, reported activation barriers as high as $4.8 \mathrm{eV}[26,27]$. Assuming that the diffusivity is thermally activated with an attempt frequency for jumping approximated by the Debye frequency of $\mathrm{SiC}$, the above range of activation energies corresponds to an error bar in the annealing temperature of nearly $600^{\circ} \mathrm{C}$.

The above discussion relates to $V_{\mathrm{C}}$ diffusivity in $3 \mathrm{C}-\mathrm{SiC}$. However, regarding $4 \mathrm{H}-\mathrm{SiC}$ (the material of choice by the industry to fabricate power devices), we are dealing with a problem which is largely unchartered. Recently, activation barriers for the jumping of point defects in $4 \mathrm{H}-\mathrm{SiC}$ (mostly concerning the $V_{\mathrm{Si}}$ defect) were shown to depend substantially on the sublattice location of the starting and ending structures [28]. A subsequent study demonstrated a similar effect for neutral $V_{C}$ [29]. Interestingly, and although not reported by the authors, from their results we can infer that $V_{C}$ diffusivity could be anisotropic. Hence, the large scattering in the reported theoretical barriers, as well as a poor understanding of the connection between crystalline anisotropy and vacancy diffusivity, call for a close look at this problem.

A deep level transient spectroscopy (DLTS) study indicated that $V_{\mathrm{C}}$ could diffuse laterally in $c$-cut (0001) samples already at room temperature [30]. This was, however, put into question in a recent experimental study, where we found that temperatures above $1200{ }^{\circ} \mathrm{C}$ [31] are required for significant $V_{\mathrm{C}}$ migration along both the $a$ and the $c$ directions $(\langle 11 \overline{2} 0\rangle$ and $\langle 0001\rangle$, respectively). In the same study, $V_{\mathrm{C}}$ diffusion in $4 \mathrm{H}-\mathrm{SiC}$ was demonstrated to be anisotropic [31]. This was achieved by combining heat treatments up to $1400^{\circ} \mathrm{C}$ with subsequent DLTS depth profiling to resolve $V_{\mathrm{C}}$ depth distributions before and after diffusion [31]. The defect was found to diffuse much farther along the basal direction (in comparison to the axial direction), but unfortunately activation energies and diffusivities for $V_{\mathrm{C}}$ migration were not reported at the time due to lack of data. Still, the small $(\sim 0.02 \%)$ difference between basal and pseudoaxial jump lengths in $4 \mathrm{H}-\mathrm{SiC}$ can hardly explain that observation. In Ref. [31] it was suggested that the anisotropy could be related to the fact that $4 \mathrm{H}$ $\mathrm{SiC}$ holds two distinct carbon vacancies, namely $V_{\mathrm{C}}(k)$ and $V_{\mathrm{C}}(h)$, but the argument was not sufficiently developed as to substantiate such a statement.

Anisotropic diffusivity can lead to inhomogeneities in the $V_{\mathrm{C}}$ concentration across SiC-based devices, degrading carrier lifetime in certain areas which are then rendered useless. Such problems are likely to be extended to dopant activation and distribution, which depend on vacancy diffusion, thus prompting a search for the details behind anisotropy during thermally activated $V_{\mathrm{C}}$ migration. In the present study we aim at elucidating the atomistic aspects of $V_{\mathrm{C}}$ migration in $3 \mathrm{C}$ - and $4 \mathrm{H}-\mathrm{SiC}$ by combining theory and experiments while taking the duality of lattice sites in $4 \mathrm{H}-\mathrm{SiC}$ into account. To that end, we employ hybrid (nonlocal) and semilocal density functional calculations, combining the nudged elastic band and dimer methods to explore the potential energy surface along individual vacancy jumps. Moreover, we study thermally activated $V_{\mathrm{C}}$ migration experimentally (in $4 \mathrm{H}-\mathrm{SiC}$ only), by combining heat treatments and DLTS depth profiling measurements, and quantify $V_{\mathrm{C}}$ diffusivities along two different crystallographic directions: the $c$ direction and the $a$ direction. In addition to yielding the energy barriers for $V_{\mathrm{C}}$ migration, the experimental data offers the possibility to determine the mechanism by which carbon vacancies migrate in $4 \mathrm{H}-\mathrm{SiC}$.

The paper is organized in the following way. Section II contains the relevant computational details, with a large portion of this section devoted to tests and benchmarking to support our results. The experimental methods used are also found in Sec. II. The main findings of this work are presented in Sec. III, divided into a theory section (which is separated according to the $\mathrm{SiC}$ polytype under scrutiny) and an experimental section. Section IV is devoted to a comparison of the theoretical and experimental results, a discussion of 
key issues including the electronic and atomistic structure along migration paths, and consequences for self-diffusion in $4 \mathrm{H}-\mathrm{SiC}$. Finally, concluding remarks are provided in Sec. V.

\section{METHODS}

\section{A. Theory \\ 1. Computational details}

All defect calculations were performed using the VASP density functional software [32-35], with which we found electronic ground states within the Kohn-Sham formalism, using the projector augmented-wave (PAW) method [36] and plane waves to describe core and valence electrons, respectively. Supercells of $3 \mathrm{C}-\mathrm{SiC}$ (4H-SiC) with cubic (hexagonal) shape were constructed by replicating $3 \times 3 \times 3(5 \times 5 \times 2)$ conventional (primitive) cells along the main axes. Pristine supercells comprised a total of 216 (400) atoms, from which one carbon atom was removed to create a $V_{\mathrm{C}}$ defect. The lattice constants were $a_{0}=4.347 \AA$ for $3 \mathrm{C}-\mathrm{SiC}$, while for $4 \mathrm{H}-\mathrm{SiC}$ we used $a=3.071 \AA$ and $c=10.152 \AA$. These figures were obtained within screened hybrid density functional theory (HSE06 [37,38]), upon relaxation of primitive cells using fully converged $13 \times 13 \times 13$ - and $13 \times 13 \times 7-\Gamma$-centered grids of $\mathbf{k}$ points to sample the Brillouin zone (BZ), respectively. While differing by less than $0.6 \%$ from their experimental counterparts $[39,40]$, such a discrepancy is not expected to induce significant effects to the calculated migration barriers.

All $V_{\mathrm{C}}$ ground state geometries were relaxed within the generalized gradient approximation to the exchange correlation potential as proposed by Perdew, Burke, and Ernzerhof (PBE) [41]. The plane-wave energy cutoff was set to $420 \mathrm{eV}$. Brillouin zones of $3 \mathrm{C}$ - and $4 \mathrm{H}-\mathrm{SiC}$ supercells were sampled with $2 \times 2 \times 2$ Monkhorst-Pack and $2 \times 2 \times 2 \Gamma$-centered k-point meshes, respectively. The electronic self-consistent energy was minimized to a numerical accuracy of $10^{-6} \mathrm{eV}$, while atomic coordinates of stable structures were relaxed by means of a conjugate-gradient algorithm until the largest force was lower than $0.01 \mathrm{eV} / \AA$. The resulting defect structures matched recent semilocal and hybrid DFT calculations $[14,19,42]$.

To investigate individual jumps between different lattice sites during $V_{\mathrm{C}}$ migration, a combination of the nudged elastic band (NEB) $[43,44]$ and the dimer [45] methods was employed at the PBE level. When commencing a NEB calculation of each jump, initial and final geometries are fixed, and a chain of intermediate changeable images (like frames in a movie sequence) are created along the configurational space between the end points. The images are connected by spring interactions to avoid becoming either too close or too distant from each other. Finally, they are collectively optimized, resulting in a series of images that closely follow a minimum energy path (MEP) connecting the end structures.

In a first step, NEB calculations yielded a preliminary portrayal of the MEP for each vacancy-atom exchange step. They approximately describe the mechanism where a carbon atom neighboring the vacancy jumps into the vacant site, leaving a new $V_{\mathrm{C}}$ in its wake. For these exploratory inspections of the MEP, 11 intermediate images, a plane-wave energy cutoff of $E_{\text {cut }}=300 \mathrm{eV}$, and the $\Gamma$ point for BZ sampling were deemed sufficient. The end structures were still those obtained within the higher level of accuracy (concerning basis and BZ sampling) as employed during the relaxation of the ground state structures.

Due to the use of supercells and the concomitant BZ folding, the band gap of the Kohn-Sham electronic structure becomes direct and narrowest at $\mathbf{k}=\Gamma$. On top of that, the use of a semilocal functional, and the unavoidable underestimated gap width, affects structures and energies due to spurious over mixing of gap states (from the vacancy) with crystalline states, particularly at the $\Gamma$ point [42].

Given the above, a refinement of the calculated transitionstate structures was needed, most importantly to employ a set of BZ sampling points with lower mixing effects. For that purpose, the $\mathbf{k}$ points for BZ sampling and $E_{\text {cut }}$ were chosen to be identical to those used for the ground state relaxations. Hence, we either carried out (i) upgraded NEB calculations restricted to a narrower sequence of images enclosing the higher energy section obtained from the exploratory MEP or (ii) used a local search algorithm, namely the dimer method to find the saddle point. In both cases, the starting point was the result from the exploratory $\Gamma$-point NEB calculations. We found that either way, both methods yield approximately identical results. However, due to its lower computational cost, the dimer method was mostly used.

The dimer method requires two images comprising the actual dimer, which are displaced from each other by a small distance and should be close to the saddle point. The saddle point can then be identified by consecutive dimer rotations and changes in dimer separation within the potential energy surface [45]. Provided that we have a good starting structure, the dimer method was found to be a swift and dependable transition-state search algorithm [46].

Finally, after identification of the transition-state geometries, single-point energies of the initial, transition, and final states were determined self-consistently by using the screened hybrid density functional HSE06 [37,38], $\Gamma$ only for BZ sampling and $E_{\text {cut }}=420 \mathrm{eV}$ for the plane-wave energy cutoff. Activation energies for $V_{\mathrm{C}}$ jumps were obtained from the energy difference between transition and initial states.

The HSE06 functional is widely accepted as capable of accurately capturing the $3 \mathrm{C}$ - and $4 \mathrm{H}-\mathrm{SiC}$ electronic structure, yielding calculated band gaps of $2.24 \mathrm{eV}$ and $3.17 \mathrm{eV}$ at $T=0 \mathrm{~K}$, respectively, close to the experimental values of $2.4 \mathrm{eV}$ [11] and $3.27 \mathrm{eV}$ [47]. The use of screened hybrid DFT energies of PBE-relaxed structures was recently tested for solid-state problems, including defects in $\mathrm{SiC}$ [48]. This approach allows us (i) to employ large enough supercells to accommodate the strain fields produced by the vacancy and at the same time (ii) to avoid artificial hybridization between vacancy and crystalline states.

We may summarize the methodology employed as the following recipe (applied to both $3 \mathrm{C}$ - and $4 \mathrm{H}-\mathrm{SiC}$ ):

(1) Relaxation at PBE level, with BZ $2 \times 2 \times 2$-sampling and $E_{\text {cut }}=420 \mathrm{eV}$, to obtain initial and final $V_{\mathrm{C}}$ structures involved in the jumps.

(2) Exploratory NEB calculation at PBE level, with BZ $\Gamma$ sampling and $E_{\text {cut }}=300 \mathrm{eV}$, to identify an approximate MEP and transition state. 
(3) Transition-state search using the dimer method at PBE level, BZ $2 \times 2 \times 2$ sampling and $E_{\text {cut }}=420 \mathrm{eV}$. The initial dimer was made from the two highest energy structures from the exploratory NEB run.

(4) Total energy calculations of PBE geometries at HSE06-level, with BZ $\Gamma$ sampling and $E_{\text {cut }}=420 \mathrm{eV}$, to find the energy barriers for $V_{\mathrm{C}}$ jumps.

\section{Workflow testing}

While the method presented in the previous section was being developed, several tests were performed to ensure the accuracy and correctness of each calculation step by investigating whether the $V_{\mathrm{C}}$ migration barrier height was converged with respect to the BZ sampling at both the PBE and HSE06 levels. Moreover, several variations of the methodology were tested, to ensure that they yielded the same result. Due to the larger supercell size and greater complexity of $4 \mathrm{H}-\mathrm{SiC}$, tests were firstly performed in 3C-SiC. Final HSE06-level results for $4 \mathrm{H}-\mathrm{SiC}$ will be presented in Sec. III. However, some benchmarking tests are also included herein for reference.

Prior to testing barrier height convergence with respect to k-point sampling, the credibility of the method needed to be verified. Following an 11-image $\Gamma$-NEB calculation to obtain an exploratory $\mathrm{MEP}$ of $V_{\mathrm{C}}$ in $3 \mathrm{C}-\mathrm{SiC}$, we performed a second and more focused NEB calculation using a $2 \times 2 \times 2$ mesh of $\mathbf{k}$ points and seven images distributed along a short configurational segment enclosing the saddle point region of the exploratory MEP. A comparison between the activation energy for migration, $E_{\mathrm{A}}$, obtained according to the twofold NEB calculation described above and that obtained from the dimer approach, is shown in Table I. We conclude that both methods essentially lead to the same result, $E_{\mathrm{A}}=3.7 \mathrm{eV}$. Table I also shows that the simpler exploratory NEB calculation (first data row) provides a barrier which is only $\sim 0.2 \mathrm{eV}$ below the best result, suggesting that the highest energy image is indeed a good starting point for the subsequent methods.

We also verified the BZ-sampling accuracy of a $2 \times 2 \times$ 2 k-point sampling mesh. The test was carried out using seven-image focused NEB runs as described above. An identical calculation with a $4 \times 4 \times 4 \mathbf{k}$-point mesh gave an energy

TABLE I. Test of the transition state optimization method in 3CSiC. First row: Exploratory MEP search using a $\Gamma$-point 11 -image NEB calculation. Second row: Focused 7-image NEB calculation over a short configurational segment of the exploratory MEP, near the saddle point, using $2 \times 2 \times 2 \mathrm{BZ}$ sampling. Third row: Dimer run for identifying the saddle point structure. Fourth row: Focused 7-image NEB calculation near the saddle point, using $4 \times 4 \times 4 \mathrm{BZ}$ sampling. All calculations were done at the PBE level with $E_{\text {cut }}=300 \mathrm{eV}$ for the $\Gamma$-NEB and $E_{\text {cut }}=420 \mathrm{eV}$ for the remaining calculations.

\begin{tabular}{lccc}
\hline \hline Method & Functional & k-mesh & $E_{\mathrm{A}}(\mathrm{eV})$ \\
\hline NEB (11-img) & PBE & $\Gamma$ & 3.52 \\
NEB (7-img) & PBE & $2 \times 2 \times 2$ & 3.66 \\
Dimer & PBE & $2 \times 2 \times 2$ & 3.69 \\
NEB (7-img) & PBE & $4 \times 4 \times 4$ & 3.66 \\
\hline \hline
\end{tabular}

TABLE II. Convergence testing of $V_{\mathrm{C}}$ migration barrier heights in $3 \mathrm{C}$ - and $4 \mathrm{H}-\mathrm{SiC}$ with respect to $\mathrm{BZ}$ sampling when using the two-step method Dimer+SCF@HSE06, namely a PBE-level dimer search (employing a MP- or $\Gamma$-centered $2 \times 2 \times 2 \mathbf{k}$ mesh) followed by a SCF (self-consistent field) calculation at the HSE06 level. In 4H$\mathrm{SiC}$, one in-plane jump [namely $h h$, standing for $\left.V_{\mathrm{C}}(h) \rightarrow V_{\mathrm{C}}(h)\right]$ and two out-of-plane jumps ( $h k$ and $k h$ ) were investigated. Coordinates of $\mathbf{k}$ points are in units of reciprocal lattice vectors. All values are in $\mathrm{eV}$.

\begin{tabular}{lcccc}
\hline \hline & & \multicolumn{3}{c}{$4 \mathrm{H}-\mathrm{SiC}$} \\
\cline { 3 - 5 } BZ sampling & $3 \mathrm{C}-\mathrm{SiC}$ & $h h$ & $h k$ & $k h$ \\
\hline$\Gamma=(0,0,0)$ & 3.97 & 3.68 & 4.06 & 4.17 \\
$R=\left(\frac{1}{2}, \frac{1}{2}, \frac{1}{2}\right)$ & 4.03 & & & \\
$A=\left(0,0, \frac{1}{2}\right)$ & & 3.68 & 4.05 & 4.17 \\
$\mathrm{MP}-2 \times 2 \times 2$ & 4.07 & & & \\
$\Gamma-2 \times 2 \times 2$ & & & 4.11 & \\
\hline \hline
\end{tabular}

barrier which differed by less than $2 \mathrm{meV}$ from the analogous quantity obtained with a coarser $2 \times 2 \times 2$ mesh.

The energy barrier convergence with respect to BZ sampling was investigated in $3 \mathrm{C}-\mathrm{SiC}$ when employing the dimer method followed by a final HSE06-level self-consistent field (SCF) calculation. The results of the test are summarized in Table II, including similar tests performed for the $4 \mathrm{H}-\mathrm{SiC}$ case. In $3 \mathrm{C}$-SiC, we find that the $\Gamma$-sampling result is already very close to that using a single k-point at the corner of the $\mathrm{BZ}$, namely $R=\left(\frac{1}{2}, \frac{1}{2}, \frac{1}{2}\right)$, or even using the most accurate $2 \times 2 \times 2$ mesh. The difference between the $\Gamma$ - and $2 \times 2 \times$ 2-sampled results is only $0.1 \mathrm{eV}$, the same as the expected error bar of the method.

In $4 \mathrm{H}-\mathrm{SiC}$, single-point sampling tests, namely $\Gamma$ and $A=$ $\left(0,0, \frac{1}{2}\right)$, were performed for in-plane $(h h)$ and out-of-plane ( $h k$ and $k h$ ) vacancy jumps. The difference to the $\Gamma$-centered $2 \times 2 \times 2$ result was actually smaller, down to about $0.05 \mathrm{eV}$. Accordingly, the method of choice (Dimer+SCF@HSE06) with $\Gamma$-only sampling at the HSE06 level is deemed sufficient for capturing the energetics of $V_{\mathrm{C}}$ migration in $3 \mathrm{C}$ - and $4 \mathrm{H}-\mathrm{SiC}$.

\section{B. Experiment}

The experimental section of this work concerns $4 \mathrm{H}-\mathrm{SiC}$ only as, to the best of our knowledge, no DLTS signal has been attributed to the carbon vacancy in 3C-SiC. Epitaxial layers $(\sim 10 \mu \mathrm{m})$ of $\mathrm{n}$-doped (nitrogen) $4 \mathrm{H}-\mathrm{SiC}$ with two different surface orientations were used: $c$-cut samples grown $4^{\circ}$ off the $c$ axis purchased from Cree Inc., and $a$-cut samples grown on-axis by chemical vapor deposition at $1650^{\circ} \mathrm{C}$ at the University of Linköping [49]. The samples were implanted with $4.0 \mathrm{MeV} \mathrm{C}$ ions to fluences of either $4 \times 10^{8} \mathrm{~cm}^{-2}$ or $6 \times 10^{8} \mathrm{~cm}^{-2}$ and having a projected range of $\sim 2.5 \mu \mathrm{m}$ according to collision Monte Carlo calculations as manifested in the SRIM (stopping and range of ions in matter) code [50]. All implantations were performed at room temperature with the samples tilted $\sim 8^{\circ}$ off with respect to the surface normal to reduce channeling. Carbon implantation was chosen to ensure selective formation of point defects while avoiding 
non-native species, and the implantation energy was chosen for the projected range to be within the DLTS probing region. Following ion implantation, metastable peaks tend to appear near the $\mathrm{Z}_{1 / 2}$ level in $4 \mathrm{H}-\mathrm{SiC}$ DLTS spectra [51]. Hence, a post-implantation annealing at $200^{\circ} \mathrm{C}$ was conducted to alleviate implantation damage and ensure that the $V_{\mathrm{C}}$ was primarily being addressed. Measurements after this initial annealing step are labeled prediffusion. Heat treatments were carried out at temperatures in the $200-1600{ }^{\circ} \mathrm{C}$ range, where the low temperature anneals between $200{ }^{\circ} \mathrm{C}$ and $600^{\circ} \mathrm{C}$ were performed for $0.5 \mathrm{~h}$ in air using a conventional tube furnace, while at higher temperatures argon (Ar) atmosphere and a rf-heated furnace equipped with a graphite crucible were employed.

After the heat treatments, circular Ni Schottky contacts, having a diameter of $\sim 1 \mathrm{~mm}$ and thickness $\sim 150 \mathrm{~nm}$, were deposited on top of the epilayers using an electron-beam evaporator, whereas silver paste was used as backside Ohmic contacts. The $V_{\mathrm{C}}$ concentration as a function of depth from the surface was investigated by monitoring the $Z_{1 / 2}$ peak at $\sim 285 \mathrm{~K}$ using DLTS depth profiling employing a $640 \mathrm{~ms}^{-1}$ rate window, while keeping the temperature within $\pm 0.1 \mathrm{~K}$. The DLTS setup is described elsewhere [52]. The reverse bias was kept constant at $-10 \mathrm{~V}$, while gradually increasing the filling pulse voltage (50 ms duration) from 0 to $11 \mathrm{~V}$. DLTS depth profiling yields a DLTS signal versus voltage, which can be converted into defect concentration versus depth according to [53]

$$
N_{\mathrm{t}}(x)=-\left(\frac{q W^{2} N_{\mathrm{rb}}}{\epsilon}\right) N_{\mathrm{d}}(x) \frac{\partial(\Delta C / C)}{\partial V},
$$

where $N_{\mathrm{t}}(x)$ is the defect (trap) concentration as a function of depth from the surface, $q$ is the elementary charge, $W$ the depletion width, $N_{\mathrm{rb}}$ the concentration of ionized donors at the end of the depletion region, $\epsilon$ the semiconductor permittivity, $N_{\mathrm{d}}(x)$ the concentration of ionized donors versus depth, and the last factor is the derivative of the DLTS signal versus voltage. The ionized donor concentrations, $N_{\mathrm{rb}}$ and $N_{\mathrm{d}}(x)$, were determined from capacitance-voltage measurements that were performed prior to each individual DLTS depth profiling procedure. For all measurements, $N_{\mathrm{t}}<0.1 N_{\mathrm{d}}$. Note that Eq. (1) is a commonly used approximation which neglects the $\lambda$-correction term [54-56]. We will return to this issue in Sec. III B.

\section{RESULTS}

\section{A. Theory}

First of all, let us identify the number of possible ways a $V_{\mathrm{C}}$ defect can jump into a neighboring carbon site. Due to (pseudo-)Jahn-Teller effects, neutral $V_{\mathrm{C}}$ adopts tetragonal $\left(D_{2 d}\right)$ and monoclinic $\left(C_{1 h}\right)$ ground state structures in $3 \mathrm{C}$ - and $4 \mathrm{H}-\mathrm{SiC}$, respectively $[14,24,42,57,58]$. In the cubic phase, each carbon site has one shell of 12 carbon second neighbors, shown as black haloed spheres in Fig. 1(a). Due to symmetry lowering, a tetragonal $V_{\mathrm{C}}$ in $3 \mathrm{C}-\mathrm{SiC}$ has two distinct shells of $\mathrm{C}$ neighbors (respectively populated with $4+8=12$ atoms). After each jump the vacancy can land with up to three symmetry-equivalent orientations, meaning that in principle we would have to consider 2 (shells) $\times 3$ (orientations) $=6$ different ways of jumping. For the case of monoclinic $V_{\mathrm{C}}$ in $4 \mathrm{H}-\mathrm{SiC}$, there are two inequivalent lattice sites, each having seven inequivalent shells of carbon neighbors (respectively populated with $1+2+2+2+2+$ $2+1=12$ atoms). Given that there are three symmetryequivalent orientations of $V_{\mathrm{C}}$ for each lattice site, we end up with a total of 42 different jumps.

Fortunately, we know that the height of the energy barriers for conversion between equivalent (pseudo-)Jahn-Teller induced alignments, are about $0.3-0.4 \mathrm{eV}$ [14]. Consequently, during vacancy migration, the temperatures are high enough to allow the defect to freely roam around all orientations and effectively show tetrahedral or trigonal symmetry in $3 \mathrm{C}$ - and $4 \mathrm{H}-\mathrm{SiC}$, respectively. From the NEB runs, we actually found that during the very early stages of each jump, the $V_{\mathrm{C}}$ defect nearly gains the full symmetry, and from there, proceeds with the actual jump. Hence, we assume that the saddle point structure does not depend on the choice of initial/final orientation of the defect. This reduces the number of jumps to one in $3 \mathrm{C}-\mathrm{SiC}$ and to three for each vacancy $\left[V_{\mathrm{C}}(k)\right.$ and $\left.V_{\mathrm{C}}(h)\right]$ in $4 \mathrm{H}-\mathrm{SiC}$.

The possible jumps are depicted in Fig. 1, where curved arrows illustrate approximate paths of the carbon vacancy. In

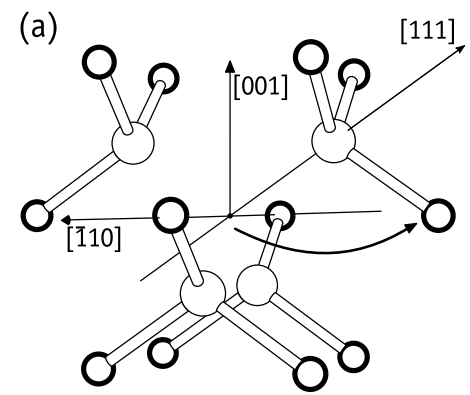

$\mathrm{V}_{\mathrm{C}}$ in $3 \mathrm{C}-\mathrm{SiC}$

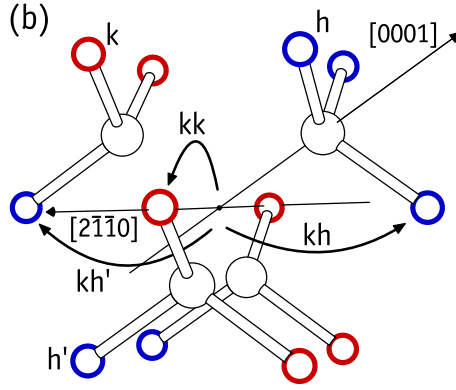

$\mathrm{V}_{\mathrm{C}}(\mathrm{k})$ in $4 \mathrm{H}-\mathrm{SiC}$

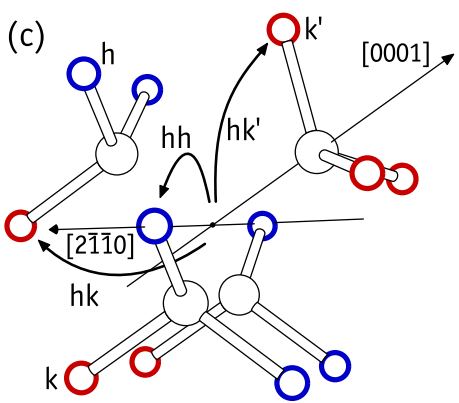

$\mathrm{V}_{\mathrm{C}}(\mathrm{h})$ in $4 \mathrm{H}-\mathrm{SiC}$

FIG. 1. Illustration of the possible jumps of (a) $V_{\mathrm{C}}$ in $3 \mathrm{C}-\mathrm{SiC}$, (b) $V_{\mathrm{C}}(k)$ in $4 \mathrm{H}-\mathrm{SiC}$, and (c) $V_{\mathrm{C}}(h)$ in $4 \mathrm{H}-\mathrm{SiC}$, into nearest neighbor C sites. $\mathrm{Si}$ and $\mathrm{C}$ atoms are represented in white, with thin and thick halos, respectively. Red- and blue-circled atoms in $4 \mathrm{H}-\mathrm{SiC}$ differentiate $\mathrm{C}$ atoms occupying $k$ and $h$ planes. Curved arrows illustrate approximate paths of the carbon vacancy and point to the $\mathrm{C}_{\mathrm{x}}$ atom of that particular jump (see text). Individual jumps are labeled by letter pairs indicating initial and final lattice sites of $V_{\mathrm{C}}$. In $4 \mathrm{H}-\mathrm{SiC}, k h$ and $k h^{\prime}$ are symmetrically related to $h k$ and $h k^{\prime}$, respectively (see text). 
3C-SiC all $V_{\mathrm{C}}$ jumps are symmetrically equivalent, leaving a single jump (among 12 possibilities) to be explored. It is convenient at this point to single out the shell of twelve carbon atoms directly bound to the $\mathrm{Si}$ atoms that are edging the vacancy. During a vacancy jump, any of these $\mathrm{C}$ atoms can exchange their position with the vacancy. Hereafter, the jumping $\mathrm{C}$ atom is labeled $\mathrm{C}_{\mathrm{x}}$, standing for exchanging $C$ atom. Analogously, the shell of twelve carbon atoms is referred to as $\mathrm{C}_{\mathrm{x}}$ shell.

In Figs. 1(b) and 1(c), individual jumps in $4 \mathrm{H}-\mathrm{SiC}$ are labeled by letter pairs indicating initial and final lattice sites of $V_{\mathrm{C}}$. Pseudocubic and hexagonal carbon sites are highlighted with red and blue halos, respectively. For each lattice site we have one basal jump $\left(k k^{\prime}\right.$ and $\left.h h^{\prime}\right)$ and two pseudoaxial jumps, ${ }^{1}$ namely $\left\{k h, k h^{\prime}\right\}$ for $V_{\mathrm{C}}(k)$ and $\left\{h k, h k^{\prime}\right\}$ for $V_{\mathrm{C}}(h)$, respectively. We also note that, if $h^{\prime}-k-h-k^{\prime}$ stands for the sequence of four carbon atoms in the $4 \mathrm{H}-\mathrm{SiC}$ primitive cell along the axial direction, $k h$ is related to $h k$ by reversal symmetry, and in the same way, $k h^{\prime}$ is a reversed $h k^{\prime}$ jump. So, the irreducible number of jumps to consider in $4 \mathrm{H}-\mathrm{SiC}$ is four. Two of them span a basal lattice vector $(\{k k, h h\})$, while the other two, for instance $\left\{k h^{\prime}, k h\right\}$ span the axial vector along a $-h^{\prime}-k-h-k^{\prime}$ - chain of sites.

\section{1. $3 \mathrm{C}-\mathrm{SiC}$}

As reported previously in Ref. [24], $V_{\mathrm{C}}$ in 3C-SiC migrates on the $\mathrm{C}$ sublattice over a calculated barrier of $3.5 \mathrm{eV}$. This figure was obtained using a local functional and $\Gamma$ sampling $[59,60]$, and reproduced by us (see first data row in Table I). A subsequent NEB calculation with $2 \times 2 \times 2$ BZ sampling mesh, followed by a dimer saddle-point search, raised the barrier to $3.7 \mathrm{eV}$. Despite the difference, the mechanism for the vacancy hop, as well as the transition state geometry, are essentially the same and confirm what was previously found.

Figure 2(a) shows a $C_{x}$ atom (highlighted in black) initiating its jump in NEB image number 1 . We note that NEB image numbers in the figure refer to the location of the vacancy (black dot). Both the $\mathrm{C}_{\mathrm{x}}$ atom and $V_{\mathrm{C}}$ meet at the transition state near NEB image number 5 and finally exchange their locations at the final (ground) state in NEB image number 13. The calculated exploratory MEP along the configurational space (using $\Gamma$ sampling and $E_{\text {cut }}=300 \mathrm{eV}$ ) is shown in Fig. 2(b) using a dashed line and black dots. In Ref. [24], the transition state was rationalized as the configuration at which the $\mathrm{C}_{\mathrm{x}}$ atom crosses through a gate defined by the rectangle drawn in Fig. 2(a). Although this picture is well suited for $3 \mathrm{C}-\mathrm{SiC}$, it is not general enough to be applied in $4 \mathrm{H}-$ $\mathrm{SiC}$. We prefer to describe the transition state (close to NEB image number 5) as a $\mathrm{Si}-\mathrm{C}$ split-interstitial (nearly aligned along $\langle 001\rangle)$ shouldered by two carbon vacancies. Below we argue that the height of the barrier depends mostly on the coordination of the atoms in the $\mathrm{Si}-\mathrm{C}$ unit at the saddle-point configuration, including bond lengths and angles with their ligands.

\footnotetext{
${ }^{1}$ The term pseudoaxial is employed here to emphasize that this is not a purely axial jump of $V_{\mathrm{C}}$ along a $\langle 0001\rangle$ crystallographic direction. This contrasts with basal jumps, where vacancy motion proceeds strictly within a basal plane.
}
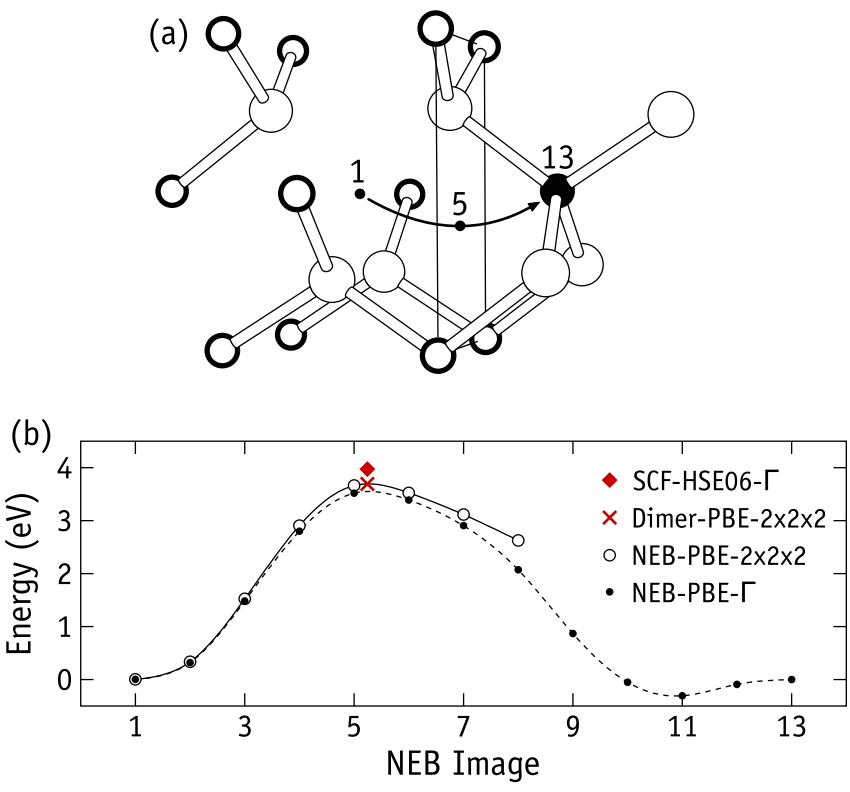

FIG. 2. $V_{\mathrm{C}}$ migration in $3 \mathrm{C}-\mathrm{SiC}$ as illustrated by (a) the atomistic structure surrounding a $V_{\mathrm{C}}$ defect ( $\mathrm{Si}$ and $\mathrm{C}$ shown with thin and thick halos, respectively) and the approximate path a $\mathrm{C}_{\mathrm{x}}$ atom (shown in black) will follow when jumping into the vacant site, in the opposite direction of the curved arrow. Dots and numbers indicate the position of the $V_{\mathrm{C}}$ and respective NEB images along the MEP. In (b) we show the energies of intermediate structures along the MEP, as obtained using the NEB method with different BZ sampling meshes (dots and open symbols), using the dimer method (red cross), and performing a SCF calculation within hybrid-DFT using the structure identified by the dimer search (red diamond).

The below-zero dip towards the end of the MEP curve of Fig. 2(b) (close to NEB image number 11) is an artifact. It arises because the end structures (which are not changed during the NEB relaxation) were obtained using a denser BZ sampling mesh and a higher plane-wave cutoff energy than the NEB calculation. Structures from NEB images number 11 and 13 are rather close in configurational space. However, the former is closer to the ground state when using $\Gamma$ sampling and $E_{\text {cut }}=300 \mathrm{eV}$.

Figure 2(b) also shows transition state energies calculated (i) using a more accurate NEB run focused on a shorter path near the saddle point (NEB-PBE- $2 \times 2 \times 2$, solid line and open symbols), (ii) using the dimer method (Dimer-PBE$2 \times 2 \times 2$, red cross), and (iii) using the structure from the dimer search to perform an SCF calculation at the HSE06level (SCF-HSE06- $\Gamma$, red diamond). While both PBE-level calculations give $E_{\mathrm{A}}=3.7 \mathrm{eV}$, the hybrid-DFT result is $E_{\mathrm{A}}=$ $4.0 \mathrm{eV}$, significantly increasing the calculated barrier with respect to the $3.5 \mathrm{eV}$ previously found [24].

As referred by Rauls et al. [26], the total energy of a defect (and consequently its migration barrier) is sensitive to the energetic position of the occupied defect levels within the band gap. Our results indicate that improving the level of theory from local or semilocal DFT to hybrid density functional theory with a converged Brillouin-zone sampling grid, opens up the calculated gap of $\mathrm{SiC}$, decreases the coupling between defect-levels and crystalline states, and for the case of $V_{\mathrm{C}}$ the 
TABLE III. Activation energies, $E_{\mathrm{A}}$, for all four inequivalent jumps of $V_{\mathrm{C}}$ in $4 \mathrm{H}-\mathrm{SiC}$ via exchange with carbon neighbors, see Figs. 1(b) and 1(c). The three data columns refer to the three steps of the calculation, namely (1) the exploratory NEB run, (2) the dimer run with stringent $\mathrm{BZ}$ sampling mesh and $E_{\text {cut }}$, and (3) the SCF calculation of the transition state within HSE06. All activation energies for individual jumps are calculated with respect to the energy of the initial state. The overall barriers for axial and basal migration are also reported. Relevant computational details are listed in the bottom part of the table.

\begin{tabular}{lccc}
\hline \hline Jump & \multicolumn{3}{c}{ Activation energy, $E_{\mathrm{A}}(\mathrm{eV})$} \\
\hline$k h^{\prime}$ & 3.5 & 3.9 & 4.2 \\
$k h$ & 3.3 & 3.8 & 4.1 \\
$k k$ & 3.3 & 3.7 & 4.0 \\
$h h$ & 3.0 & 3.4 & 3.7 \\
Axial barrier & 3.5 & 3.9 & 4.2 \\
Basal barrier & 3.0 & 3.4 & 3.7 \\
Calculation & NEB & Dimer & SCF \\
Functional & PBE & PBE & HSE06 \\
$E_{\text {cut }}(\mathrm{eV})$ & 300 & 420 & 420 \\
BZ-sampling & $\Gamma$ & $2 \times 2 \times 2$ & $\Gamma$ \\
\hline \hline
\end{tabular}

barrier suffers an enhancement of about $0.5 \mathrm{eV}$. This energy cannot be interpreted as a source of error and is not negligible.

\section{2. $4 \mathrm{H}-\mathrm{SiC}$}

The agreement between our calculations for $V_{\mathrm{C}}$ migration in $3 \mathrm{C}-\mathrm{SiC}$ and previous works [24] provides a reassuring benchmark for the following and more involved calculations in $4 \mathrm{H}-\mathrm{SiC}$. Table III summarizes the calculated activation energies for single $V_{\mathrm{C}}$ jumps in $4 \mathrm{H}-\mathrm{SiC}$ after each of the three calculation steps (exploratory NEB, dimer, and HSE06SCF, respectively), clearly demonstrating an influence of the initial/final lattice sites on the barrier height. According to our previous analysis, four jumps were considered: two pseudoaxial $\left(k h^{\prime}\right.$ and $\left.k h\right)$ and two basal ( $h h$ and $\left.k k\right)$. The notation found in Table III is the same as that of Fig. 1, describing how a vacancy moves from a particular site into neighboring sites. We also note that for the pseudoaxial jumps (first two data rows) the barrier for the reversed jump is readily obtained by subtracting $E(h)=0.1 \mathrm{eV}$, which is the energy of $V_{\mathrm{C}}(h)$ with respect to $V_{\mathrm{C}}(k)$, to the reported barriers.

Comparing basal to axial activation barriers we conclude that $V_{\mathrm{C}}$ migration in $4 \mathrm{H}-\mathrm{SiC}$ exhibits substantial anisotropy. Furthermore, we find that there are considerable differences when we compare the barriers for basal migration within $k$ and $h$ planes. The difference between activation energies of pseudoaxial and basal jumps persists across all three computational steps (NEB, dimer, hybrid-DFT SCF calculations). However, as we improve the calculation specifications and the calculated band gap width opens up, the barriers are progressively raised.

For axial migration, the relevant barrier is the highest with respect to the ground state $V_{\mathrm{C}}(k)$, namely $E_{\mathrm{A}}\left(k h^{\prime}\right)=4.2 \mathrm{eV}$. For basal migration, we have to consider that for irradiationinduced $V_{\mathrm{C}}$ populations at $1400^{\circ} \mathrm{C}$, the populations of $V_{\mathrm{C}}(k)$ and $V_{\mathrm{C}}(h)$ are comparable, and in this case the faster species

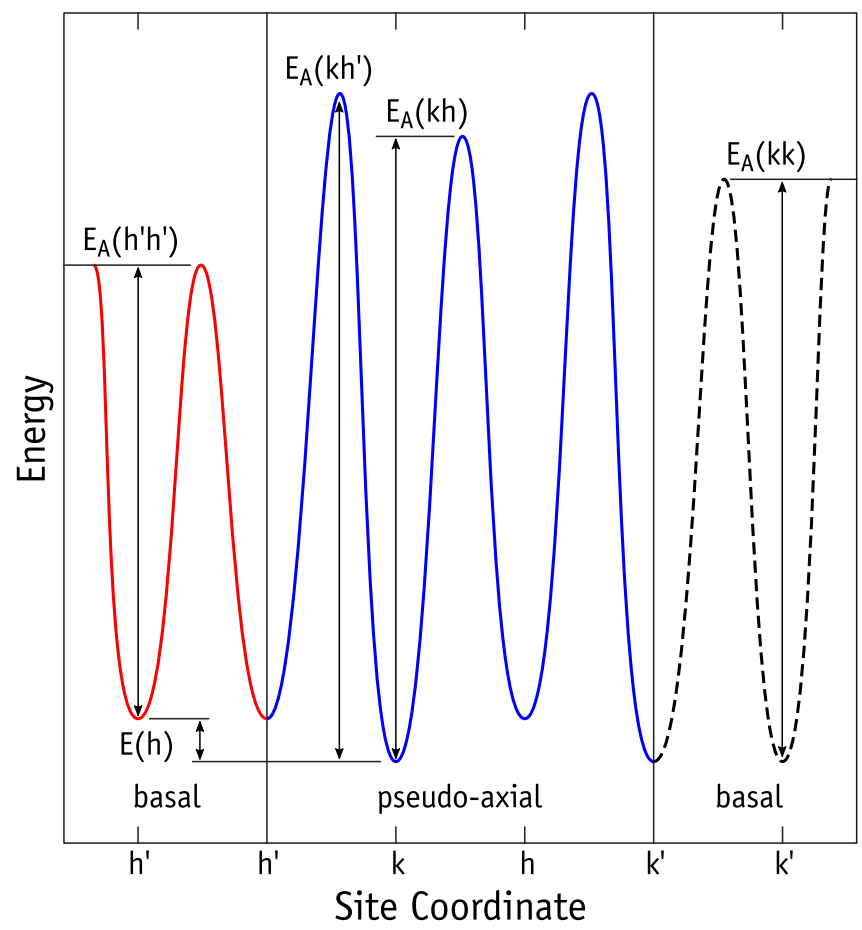

FIG. 3. Schematic potential energy profile along axial $\left(h^{\prime}-k-h-k^{\prime}\right)$ and basal $\left(h^{\prime}-h^{\prime}\right.$ and $\left.k^{\prime}-k^{\prime}\right)$ migration paths of $V_{\mathrm{C}}$ in $4 \mathrm{H}-\mathrm{SiC}$. The diagram was constructed based on the results of Table III, combined with the calculated energy of $V_{\mathrm{C}}(h)$ with respect to $V_{\mathrm{C}}(k), E(h)=$ $0.1 \mathrm{eV}$. The qualitative character of the profiles is not affected by the level of theory employed (NEB, dimer or HSE06-SCF).

will set the pace. Hence, the barrier to consider is the lowest, $E_{\mathrm{A}}(h h)=3.7 \mathrm{eV}$. The migration along the $k$ plane is limited by a barrier of $E_{\mathrm{A}}(k k)=4.0 \mathrm{eV}$, which is essentially identical to that found for the cubic phase. An overall picture of the migration of $V_{\mathrm{C}}$ in $4 \mathrm{H}-\mathrm{SiC}$ is provided in Fig. 3. The figure shows a schematic potential energy profile along axial $\left(h^{\prime}-k-h-k^{\prime}\right)$ and basal $\left(h^{\prime}-h^{\prime}\right.$ and $\left.k^{\prime}-k^{\prime}\right)$ migration paths of $V_{\mathrm{C}}$ in $4 \mathrm{H}-\mathrm{SiC}$. The blue and red lines rationalize the potential energy surface for effective axial and basal migration, whereas the dashed black line indicates that migration along the $k$-plane should be inactive. These results explain what has been previously observed in Ref. [31] but not accounted for by a physical model at the time.

Axial migration of $V_{\mathrm{C}}$ in $4 \mathrm{H}-\mathrm{SiC}$ was experimentally found to be substantially slower than basal migration [31]. At $1400^{\circ} \mathrm{C}$ they differ by about one order of magnitude [31], corresponding to a difference in activation energy of $\sim 0.3 \mathrm{eV}$. Here we are assuming identical exponential prefactors (which account for the jump attempt frequencies). This figure is in excellent agreement with the calculated $E\left(k h^{\prime}\right)-E(h h)=$ $0.5 \mathrm{eV}$ as reported in Table III. Further details regarding more recent and comprehensive experimental observations will be provided in the next section.

Interestingly, the calculated anisotropy is pronounced for $h$-basal and not so much for $k$-basal migration (compared to axial migration). As pointed out already, the activation barrier for $k$-basal migration, $E_{\mathrm{A}}(k k)=4.0 \mathrm{eV}$, is identical to the barrier for $V_{\mathrm{C}}$ migration in $3 \mathrm{C}-\mathrm{SiC}$, suggesting that the 
resulting anisotropy could be explained based on differences between the hexagonal and cubic crystal fields or bond coordination at the respective transition states within the $k$ and $h$ planes of carbon atoms. We will come back to this issue in Sec. IV. From a practical point of view, knowledge of how basal migration is activated and proceeds preferentially along $h$ planes could shape device processing techniques. For instance, deployment of impurities or dopants at a preferential site could be leveraged by interactions with either $V_{\mathrm{C}}(k)$ or $V_{\mathrm{C}}(h)$ defects, eventually biased by selective diffusion.

In a recent report by Kuate Defo et al. [29], although the diffusivity of $V_{\mathrm{C}}$ was not addressed, the energy barrier for a $k h^{\prime}$ jump of $V_{\mathrm{C}}$ in $4 \mathrm{H}-\mathrm{SiC}$ was found to be as high as $E_{\mathrm{A}} \approx 4.7-4.8 \mathrm{eV}$ using a semilocal approximation to DFT. This result is actually $\sim 0.5 \mathrm{eV}$ above our hybrid-DFT result, in opposition to the trend displayed by the semilocal results for $V_{\mathrm{C}}$ migration in $3 \mathrm{C}-\mathrm{SiC}$. For the same barrier, our semilocal calculation using the nudged elastic band + dimer method gives $E_{\mathrm{A}}=3.9 \mathrm{eV}$ (cf. $k h^{\prime}$ jump in Table III), which in turn agrees with the referred trend. We are unable to explain this inconsistency and can only suggest that the transition-state geometries from Ref. [29] were away from the true saddle point for being obtained by classical molecular dynamics.

Until now we have not mentioned the impact of entropy on the diffusivity of $V_{\mathrm{C}}$. An account on this problem was previously reported in Ref. [26]. Based on the vibrational spectrum of $V_{\mathrm{C}}$ in $3 \mathrm{C}-\mathrm{SiC}$ at the ground and transition states, vibrational entropy was found to lower the barrier by about $1.9 k_{\mathrm{B}} \mathrm{eV} / \mathrm{K}$, where $k_{\mathrm{B}}$ stands for the Boltzmann constant. From a practical point of view, this translates into a decrease of the barriers by $0.1-0.3 \mathrm{eV}$ at $1100-1600^{\circ} \mathrm{C}$. While it is important to bear in mind that these values and the experimental/theoretical error bars are close in magnitude, it is equally important to note that to some extent entropy effects will affect all barriers, irrespective of the polytype or jump type.

All four relevant MEPs for $V_{\mathrm{C}}$ migration in $4 \mathrm{H}-\mathrm{SiC}$ are shown in Fig. 4. Each chain of points on each plot (including solid and open symbols) refer to image energies from a NEB run using exploratory conditions ( $\Gamma$ sampling of the BZ and $E_{\text {cut }}=300 \mathrm{eV}$ ). The solid symbols at the ends are distinguished because they refer to single-point energy calculations employing ground state structures previously obtained using production conditions $\left(2 \times 2 \times 2\right.$ sampling and $\left.E_{\text {cut }}=420 \mathrm{eV}\right)$. Hence, likewise Fig. 2, during the NEB runs the structures edging the fixed ends relaxed towards the lowest energy configurations under exploratory conditions, which differ slightly from the true ground states (end points).

Figures 4(a) and 4(b) refer to jumps of $V_{\mathrm{C}}(k)$ into neighboring $h$ sites. Note that $k h^{\prime}$ and $k h$ plots should be read from right to left and from left to right, respectively. Both jumps (or their symmetric reversals) are performed during axial migration. They are depicted by curved arrows in Fig. 1(b). Clearly, $k h^{\prime}$ and $k h$ jumps have distinct energy profiles and expectingly different mechanisms. Conversely, $k k$ and $h h$ jumps show energy profiles close to that of $k h$, foreshadowing similar mechanisms for all three. It is also interesting that from the exploratory NEB calculations we can already infer that $k h^{\prime}$ is the highest barrier and therefore the limiting jump for axial migration. Conversely, $h h$ has the lowest barrier and should be

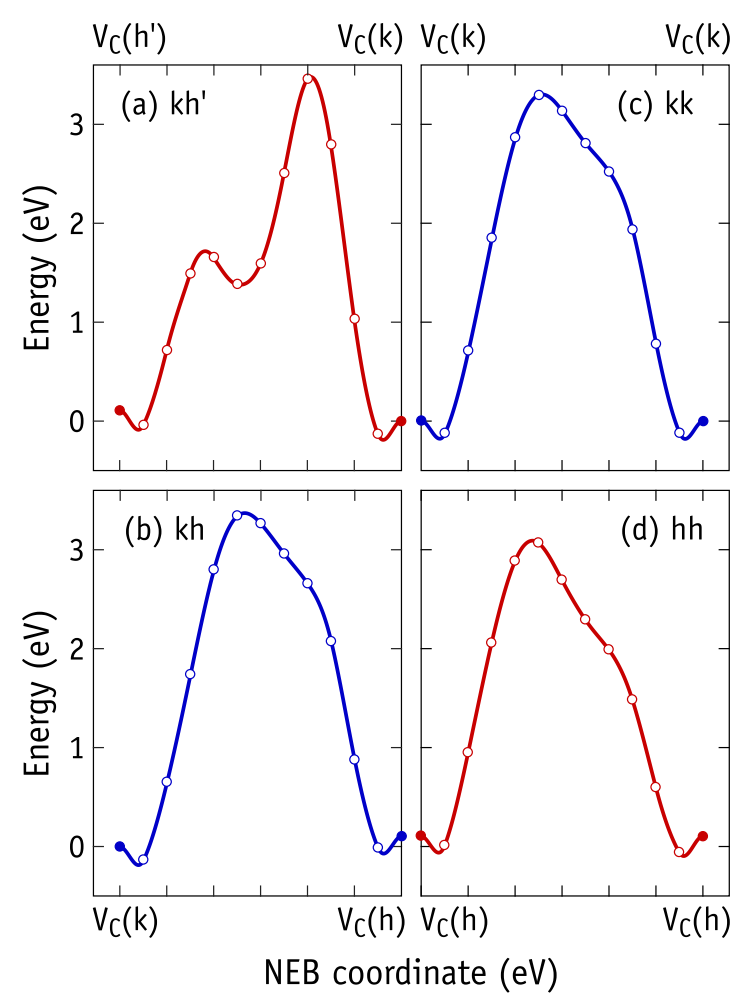

FIG. 4. Minimum energy paths (MEPs) for single jumps of $V_{\mathrm{C}}$ in $4 \mathrm{H}-\mathrm{SiC}$. (a) $k h^{\prime}$-jump, (b) $k h$-jump, (c) $k k$-jump, and (d) $h h$-jump. Calculations represented as open symbols were carried out using exploratory conditions, namely $\Gamma$ sampling of the $\mathrm{BZ}$ and $E_{\text {cut }}=$ $300 \mathrm{eV}$. Solid symbols at the ends represent ground state structures obtained using $2 \times 2 \times 2$ sampling and $E_{\text {cut }}=420 \mathrm{eV}$. Red curves indicate the MEPs which limit the diffusivity of $V_{\mathrm{C}}$ along axial and basal directions.

the limiting step regarding the diffusivity along basal planes. Below we discuss the origin of these differences.

\section{B. Experiment}

Experimentally, $V_{\mathrm{C}}$ diffusion was studied using a combination of heat treatments and deep level transient spectroscopy on $\mathrm{C}$ implanted $4 \mathrm{H}-\mathrm{SiC}$ samples. Figure 5(a) shows DLTS spectra of $c$ - and $a$-cut samples implanted with $4 \times$ $10^{8} \mathrm{~cm}^{-2} \mathrm{C}$ ions, both under prediffusion conditions and after annealing the samples at $1400^{\circ} \mathrm{C}$. Several DLTS signatures are observable in Fig. 5(a), including the previously reported peaks normally labeled $S_{1}, S_{2}$, and $Z_{1 / 2}$, with activation energies of $0.4,0.71$, and $\sim 0.7 \mathrm{eV}$, respectively [61]. The latter defect is associated with the $V_{\mathrm{C}}$ and corresponds to a twofold electron emission due to a $(-2 / 0)$ transition [15]. Vacancies at hexagonal and pseudocubic lattice sites have similar energy level positions and are only distinguishable by Laplace-DLTS [62]. The $S_{1}$ and $S_{2}$ centers arise in implanted samples but are less stable than the $Z_{1 / 2}$ level, and disappear at high temperatures [61] as seen in Fig. 5(a). Similar arguments can be presented for most of the primary defects expected to arise in the samples; the Si vacancy $\left(V_{\mathrm{Si}}\right)$ is expected to transform into the carbon antisite-vacancy (CAV) pair above $400-600^{\circ} \mathrm{C}[63,64]$, which again anneals out at $900-1200^{\circ} \mathrm{C}$ 



FIG. 5. (a) DLTS spectra of $c$-cut (blue) and $a$-cut (red) n-type $4 \mathrm{H}$-SiC in the vicinity of the $\mathrm{Z}_{1 / 2}$ level under pre- and post-diffusion conditions. The spectra have been scaled for the $\mathrm{Z}_{1 / 2}$ peak size to represent the $V_{\mathrm{C}}$ concentration of the relevant sample. (b) $V_{\mathrm{C}}$ concentration vs depth profiles along the $c$ (blue) and $a$ (red) directions in prediffusion samples and after annealing at $1400{ }^{\circ} \mathrm{C}$ for $2 \mathrm{~h}$, with dots representing experimental data, dashed lines the prediffusion fits and solid lines being the diffused profiles calculated by solving the diffusion equation.

[64,65]. Carbon and silicon self-interstitials have even lower thermal stabilities than $V_{\mathrm{Si}}$ and $\mathrm{CAV}$, with migration energies close to $\sim 1 \mathrm{eV}$ [11]. Hence, the interstitials generated both from ion collisions and implantation of $\mathrm{C}$ are expected to rapidly disappear above $200^{\circ} \mathrm{C}$, and therefore their interaction with $V_{\mathrm{C}}$ is neglected herein.

Ion implantation may also create more complex defects such as antisites, divacancies, and antisite-vacancy pairs. However, the implantation fluence ensures the selective formation of point defects to concentrations well within the dilute limit. Hence, complex defects are likely to be much less abundant than $V_{\mathrm{C}}$, and with only $\sim 10^{14} \mathrm{~cm}^{-3} V_{\mathrm{C}}$ 's in the samples implanted to the highest fluence, there should be at least $\sim 100 \mathrm{~nm}$ between defects of any kind. Therefore, and in the absence of long-range Coulomb attraction, neutral $V_{\mathrm{C}}$ 's will encounter potential sinks only rarely, and any ensuing lowering of $V_{\mathrm{C}}$ concentration (caused by reactions between $V_{\mathrm{C}}$ and complexes) should be negligible. Thus, to summarize, a target temperature range exists $\left(\sim 1200-1600{ }^{\circ} \mathrm{C}\right)$, where $V_{\mathrm{C}}$ becomes mobile, standing as the main prevalent point defect, thus enabling experimental observation of its diffusion.

A quantitative conversion of the DLTS peak amplitude to concentration is strictly valid for uniform defect profiles only. Thus, depth profiling measurements are highly appropriate for the present study, and the results for the prediffused and $1400^{\circ} \mathrm{C}$ annealed samples are shown in Fig. 5(b). A striking feature of the profiles in Fig. 5(b) is the significantly lower and broader concentration profile along the basal $a$ direction (in red) compared to that of the axial $c$ direction (in blue) for the same annealing treatment. This confirms that thermally activated $V_{\mathrm{C}}$ diffusion shows considerable anisotropy as previously proposed [31].

Figure 6 displays $V_{\mathrm{C}}$ concentration versus depth profiles for (a) $c$-cut and (b) $a$-cut samples implanted with $6 \times$ $10^{8} \mathrm{~cm}^{-2} \mathrm{C}$ ions, before and after annealing at temperatures between $1200^{\circ} \mathrm{C}$ and $1500^{\circ} \mathrm{C}$. The profiles in Figs. 6(a) and 6 (b) demonstrate the temperature evolution of the $V_{\mathrm{C}}$ concentration along axial and basal crystallographic directions, respectively, and confirm the anisotropic $V_{\mathrm{C}}$ migration in $4 \mathrm{H}-\mathrm{SiC}$. For instance, after annealing at $1500{ }^{\circ} \mathrm{C}$ the $V_{\mathrm{C}}$ has clearly moved further along the $a$ direction than the $c$ direction, despite the shorter annealing time (at $1500^{\circ} \mathrm{C}$ ) for the $a$-cut samples as compared to the $c$-cut ones. As seen from the figure, higher temperatures and/or longer diffusion times are required to obtain similar diffusion profiles for the $c$-cut [Fig. 6(a)] and the $a$-cut [Fig. 6(b)] samples, demonstrating the anisotropy in $V_{\mathrm{C}}$ diffusion. The intermediate temperature $V_{\mathrm{C}}$ profiles, i.e., between $1300^{\circ} \mathrm{C}$ and $1500^{\circ} \mathrm{C}$, become gradually lower and broader as expected from Fig. 6 for both the $c$ - and $a$-cut samples, and are therefore not shown for the sake of clearness. Annealing $c$-cut and $a$-cut samples at $1600^{\circ} \mathrm{C}$ resulted in uniform $V_{\mathrm{C}}$ concentration profiles and revealed out-diffusion of the $V_{\mathrm{C}}$, most likely to the surface and possibly to other defect sinks.

Assuming that the $\mathrm{Si}$ and $\mathrm{C}$ sublattices are independent and a single diffusion mechanism prevails with no external driving force, $V_{\mathrm{C}}$ migration is equivalent to migration in a chemically homogeneous system where isolated $V_{C}$ 's migrate exclusively on the $\mathrm{C}$ sublattice. This allows $V_{\mathrm{C}}$ diffusion to be described by a diffusion equation in the form of Fick's second law [66],

$$
\frac{\partial c}{\partial t}=D \frac{\partial^{2} c}{\partial^{2} x}
$$

where $c$ denotes the concentration and $D$ is the diffusivity. Using the prediffusion fit to the experimental data as the initial $V_{\mathrm{C}}$ concentration, we deduced the $V_{\mathrm{C}}$ diffusivity $(D)$ at each temperature by solving the diffusion equation numerically and selecting the diffusivity which resulted in the lowest least mean square error between the calculated $V_{\mathrm{C}}$ concentration profile (for a given $D$ ) and the fit to the experimental diffused $V_{\mathrm{C}}$ concentration profile. The resulting diffusivities were estimated to have accuracies in the range 20-30\%, which we will return to below.

Importantly, we note that high temperature heat treatments induce surface degradation and may influence the capacitance measured by the Schottky barrier diodes, as reflected in the relatively large error bars for the diffusivity of 20-30\%. Indeed, protective surface layers such as carbon caps exist but are known to introduce carbon interstitials that may interfere with the irradiation induced vacancies [67]. Therefore, the 



FIG. 6. $V_{\mathrm{C}}$ concentration vs depth profiles along the (a) $c$ and (b) $a$ direction before (red) and after (blue and black) annealing at temperatures between $1200{ }^{\circ} \mathrm{C}$ and $1500^{\circ} \mathrm{C}$. Circular marks represent experimental data, red dashed lines are fits to the initial profiles, and solid lines are the diffused profiles calculated by solving the diffusion equation.

following assumptions and appropriate corrections were made when processing the data: (i) the ionized donor concentration does not change during diffusion, (ii) the $V_{\mathrm{C}}$ peak position does not shift during diffusion, and (iii) the amount of $V_{\mathrm{C}}$ defects contained within the depletion region remains constant during diffusion.

The calculated $V_{\mathrm{C}}$ concentrations are shown as solid lines and experimental data as circular dots in Fig. 6, while the dashed red lines represent numerical fits to the initial experimental profiles. Evidently, solving the diffusion equation for the $V_{\mathrm{C}}$ concentration [Eq. (2)] results in an excellent agreement between the experimental and calculated $V_{\mathrm{C}}$ concentration profiles, hence, adequately describes the thermally activated $V_{\mathrm{C}}$ migration. Note that the $V_{\mathrm{C}}$ concentration profiles have been shifted slightly in depth $(0.1-0.2 \mu \mathrm{m})$ and corrected to remedy the loss of accuracy caused by surface degradation at high temperatures. The correction was performed to preserve the number of carbon vacancies within the depletion region before and after the diffusion process. Note that we assume negligible out-diffusion of $V_{\mathrm{C}}$ 's below $1600^{\circ} \mathrm{C}-$ Fick's second law relies on mass conservation. The choice of Fick's second law and conservation of $V_{\mathrm{C}}$ concentration during diffusion for modeling of the diffusion process is validated by observation of essentially identical shapes for the measured and calculated concentration profiles in Fig. 6. We note that isothermal annealing of $c$-cut samples at $1400^{\circ} \mathrm{C}$ for two and four hours, revealed identical $V_{\mathrm{C}}$ diffusivities of $D=(4 \pm 1.2) \times 10^{-14} \mathrm{~cm}^{2} / \mathrm{s}$. This is the expected observation should $V_{\mathrm{C}}$ motion in $4 \mathrm{H}-\mathrm{SiC}$ be governed by independent vacancy jumps. Furthermore, the samples annealed at $1400^{\circ} \mathrm{C}$ for 2 and $4 \mathrm{~h}$ were implanted to different $\mathrm{C}$ fluences $\left(4 \times 10^{8} \mathrm{~cm}^{-2}\right.$ and $6 \times 10^{8} \mathrm{~cm}^{-2}$, respectively). The lack of temporal dependence in the $V_{\mathrm{C}}$ diffusivity indicates no transient effects with time and confirms the elementary diffusion process.

Figure 6 demonstrates that $V_{\mathrm{C}}$ diffusion is adequately described by Fick's diffusion equation. Assuming that the underlying mechanism involves a thermally activated jump, the temperature dependence of the $V_{\mathrm{C}}$ diffusivity should follow an
Arrhenius behavior according to

$$
D=D_{0} \exp \left(-E_{\mathrm{A}} / k_{\mathrm{B}} T\right)
$$

Here, $T$ is the absolute temperature, $k_{\mathrm{B}}$ is the Boltzmann constant, $E_{\mathrm{A}}$ is the activation energy for migration, and $D_{0}$ is the exponential prefactor encompassing the jump frequency. Indeed, Fig. 7 reveals a pronounced Arrhenius behavior for $V_{\mathrm{C}}$ diffusivity in the $1300-1500^{\circ} \mathrm{C}$ range along the $c$ direction and in the $1200-1500^{\circ} \mathrm{C}$ range along the $a$ direction, where circles represent deduced $D$ values as described above. In the same figure, solid lines represent linear fits to the data. Error bars ranging from 20 to $30 \%$ are included. The error in each $D$ value was estimated from the deviation between the diffusivity and the linear fit to the experimental data in Fig. 7, combined with the observed effect of choosing different differentiation methods (smoothed vs nonsmoothed) when converting the



FIG. 7. Temperature dependence of $V_{\mathrm{C}}$ diffusivity $(D)$ along the $c$ direction (blue) and $a$ direction (red), with $D$ clearly obeying Arrhenius behavior. Circular marks represent $D$ and solid lines are linear fits to the data. The extracted fitting parameters $\left(D_{0}\right.$ and $\left.E_{\mathrm{A}}\right)$ are included in the figure. 
DLTS data into concentration vs depth profiles as described by Eq. (1). From the Arrhenius plot, fitting parameters of $D_{0}=0.54 \mathrm{~cm}^{2} / \mathrm{s}$ and $E_{\mathrm{A}}=(4.4 \pm 0.3) \mathrm{eV}$ were deduced for migration along the $c$ direction, and $D_{0}=0.02 \mathrm{~cm}^{2} / \mathrm{s}$ and $E_{\mathrm{A}}=(3.6 \pm 0.3) \mathrm{eV}$ along the $a$ direction. The absolute accuracy for the exponential prefactors is estimated to be approximately an order of magnitude, but the calculated activation energies are much more accurate with error bars of $\pm 0.3 \mathrm{eV}$ along both axial directions.

As previously mentioned, Eq. (1) does not account for the transition region, or $\lambda$ effect, arising during fast DLTS pulsing [53]. The effect has been omitted in the present data due to the surface degradation mentioned above. Tests were performed to verify our approach, e.g., for the $a$-cut sample annealed at $1500^{\circ} \mathrm{C}$ for $0.5 \mathrm{~h}$, where the high temperature is potentially associated with large surface degradation. Diffusivities deduced with and without the $\lambda$ correction differed by less than $10 \%$, which is well below the estimated error bars.

We finally note that if single negatively or double negatively charged vacancies had any influence in $V_{\mathrm{C}}$ motion, eventually for being so fast diffusing as to compensate for their low concentration under intrinsic conditions, that would be reflected in the Arrhenius plot of Fig. 7. On the contrary, it is clear that $V_{\mathrm{C}}$ motion is thermally activated with rather high barriers.

\section{DISCUSSION}

Comparing the Arrhenius profiles in Fig. 7 to the theoretical calculations reported above, we can explain the experimentally observed $V_{\mathrm{C}}$ diffusion along the $c$ and $a$ directions as the result of pseudoaxial jumps between [0001] planes and basal jumps within the $h$ plane, respectively. The measured activation energies for diffusion along the $c$ and $a$ directions, respectively, $4.4 \mathrm{eV}$ and $3.6 \mathrm{eV}$, match well with the pseudoaxial and basal barriers $E\left(k h^{\prime}\right)=4.2 \mathrm{eV}$ and $E(h h)=3.7 \mathrm{eV}$, respectively. Furthermore, and although exponential prefactors were not calculated in this work, we expect $D_{0}$ to be in the $10^{-2}-10^{-3} \mathrm{~cm}^{2} / \mathrm{s}$ range for fundamental vacancy migration [66]. This interval overlaps the prefactor found for $V_{\mathrm{C}}$ migration along the $a$ direction. Along the $c$ direction the prefactor falls off the high limit of the expected range, possibly due to limitations in the experimental accuracy. It is conceivable that configurational entropy could favor axial diffusivity due to the existence of more equivalent paths joining an axial lattice vector than a basal lattice vector. For instance, the shortest path between two vacancy configurations separated by one basal vector is unique, while up to 15 equivalent shortest paths are available for a displacement along the axial lattice vector. Another effect that would be worth exploring is correlation effects taking place during the consecutive jumps needed for axial migration. To that end, kinetic Monte Carlo simulations could be helpful.

The strong correlation between experimental and theoretical values for $V_{\mathrm{C}}$ migration demonstrates that the defect travels faster along basal directions (than along the main axial direction). Combining the experimentally observed $V_{\mathrm{C}}$ diffusion characteristics with the theoretically predicted atomistic aspects of $V_{\mathrm{C}}$ migration, we arrive at the conceptual migration diagram shown in Fig. 8, which provides an interpretation of (a) c-cut sample

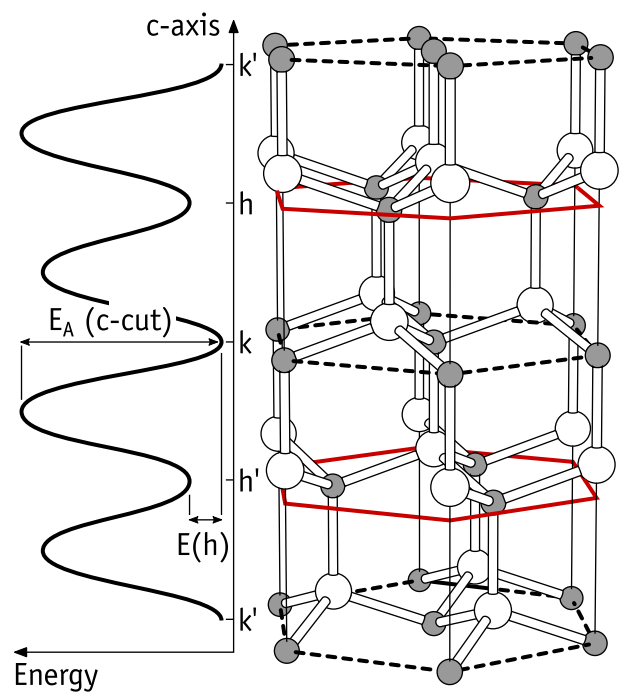

(b) a-cut sample



FIG. 8. 4H-SiC atomic structure (middle) and schematic potential energy profiles along axial (right) and basal (left) migration paths of $V_{\mathrm{C}}$ in $4 \mathrm{H}-\mathrm{SiC}$. Axial $V_{\mathrm{C}}$ migration $\left(h^{\prime} k h k^{\prime}\right)$ along the $c$ direction is shown to the left (a), and basal $V_{\mathrm{C}}$ migration ( $h h$ and $k k$ ) along the $a$ direction is pictured to the right (b). The diagram was constructed based on the results of Table III, combined with the calculated energy of $V_{\mathrm{C}}(h)$ with respect to $V_{\mathrm{C}}(k), E(h)=0.1 \mathrm{eV}$.

the experimental observables based on the theoretical model presented herein. Schematic potential energy profiles for $V_{\mathrm{C}}$ migration along the axial direction and within the $h$-basal plane [Figs. 8(a) and 8(b), respectively] are related to experimentally observed diffusivities measured in $c$-cut and $a$-cut samples, respectively. Relevant basal planes are also represented in the middle panel of Fig. 8. The shape of the potential energy profiles in Figs. 8(a) and 8(b) are representative of their relative barrier heights as reported in Table III.

Finally, we expect ion implantation to generate approximately equal amounts of $V_{\mathrm{C}}(k)$ and $V_{\mathrm{C}}(h)$ defects, overwriting whatever population ratio existed prior to implantation. Despite $V_{\mathrm{C}}(k)$ being the ground state and $V_{\mathrm{C}}(h)$ being $E(h)=$ $0.1 \mathrm{eV}$ higher in energy, the annealing temperatures employed herein $\left(\sim 1500{ }^{\circ} \mathrm{C}\right)$ are too low to drive a significant population difference by generating new carbon vacancies. Hence, lateral migration along the $h$ plane is expected to be dominant, as supported by the strong overlap between experimentally and theoretically deduced activation energies for $V_{\mathrm{C}}$ migration (3.6 eV and $3.7 \mathrm{eV}$, respectively) along the $a$ direction.

To better understand the anisotropy in $V_{\mathrm{C}}$ migration, let us have a closer look at the migration path. The carbon vacancy in $3 \mathrm{C}-\mathrm{SiC}$ migrates according to the mechanism depicted in Fig. 2 [24]. The transition state is located close to midway between two neighboring ground state structures. Near NEB images number 5 and 6 , the $\mathrm{C}_{\mathrm{x}}$ atom becomes overcoordinated (connected to five $\mathrm{Si}$ atoms), while its $\mathrm{Si}$ nearest neighbor becomes undercoordinated (connected to three $\mathrm{C}$ atoms). This departure from the stable fourfold coordination is responsible for most of the potential energy to be surmounted during the jump. 



FIG. 9. Atomistic structures of $V_{\mathrm{C}}$ in $4 \mathrm{H}-\mathrm{SiC}$ near midway between two neighboring lattice sites. Respective exploratory MEP energies are shown in Fig. 4. Si atoms are shown as large white spheres, while $\mathrm{C}_{\mathrm{x}}$-shell atoms are highlighted with red and blue halos when located in $k$ and $h$ planes, respectively. The jump of the vacancy is represented by an arrow. The $\mathrm{C}_{\mathrm{x}}$ atom is shown in black.

Figure 9 represents structures of $V_{\mathrm{C}}$ defects near midway along their MEPs according to the four different jump types that were investigated in $4 \mathrm{H}-\mathrm{SiC}$. The curved arrows indicate approximate paths of the vacancy during the jump. The $\mathrm{C}_{\mathrm{x}}$ atom (shown in the middle) moves in the opposite direction, i.e., departs from the site pointed by the arrow head.

In $4 \mathrm{H}-\mathrm{SiC}$, axial migration involves a sequence of $k h$ - and $k h^{\prime}$-like jumps. While the $k h$ MEP of Fig. 4(b) is similar both in height and shape to the MEP of $V_{C}$ in $3 \mathrm{C}$-SiC shown in Fig. 2, the $k h^{\prime}$ MEP displays an asymmetric camel back shape with a local minimum close to halfway. We can follow the $C_{x}$ atom during the $k h^{\prime}$ jump with the help of Figs. 4(a) and 9(a). Accordingly, $\mathrm{C}_{\mathrm{x}}$ departs from the $h^{\prime}$ site when $V_{\mathrm{C}}$ departs from the $k$ site. The structure quickly arrives at the saddle point, were $\mathrm{C}_{\mathrm{x}}$ passes between two $\mathrm{Si}$ atoms, becoming twofold coordinated. This is a highly unfavorable coordination for carbon and leads to the highest energy configuration along the MEP for axial migration. Then, at midway towards the $k$ site, the moving $\mathrm{C}_{\mathrm{x}}$ atom becomes fourfold coordinated as depicted in Fig. 9(a), leading to the prominent local minimum in the middle of Fig. 4(a). From here, $C_{x}$ has to overcome a small ( $\sim 0.3 \mathrm{eV}$ barrier) to reach the $k$ site, annihilating the original $V_{\mathrm{C}}(k)$ defect but creating a new $V_{\mathrm{C}}\left(h^{\prime}\right)$ defect. We note that the structure of Fig. 9(a) is a true local minimum. A free relaxation of that structure (without the spring constraints of the NEB method) did not significantly alter the geometry, and the energy of the metastable configuration was $2.11 \mathrm{eV}$ (HSE06-level) above that of the $V_{\mathrm{C}}(k)$ ground state.

The local minimum structure depicted in Fig. 9(a) (with $\mathrm{C}_{\mathrm{x}}$ showing fourfold coordination), is not visited during other vacancy jumps in $4 \mathrm{H}-\mathrm{SiC}$, namely $k h, k k$, and $h h$. There, the picture was found to be analogous to that of $3 \mathrm{C}-\mathrm{SiC}$. The transition-state structures are close to halfway between initial and final structures as depicted in Figs. 9(b)-9(d). Similarly to the transition state in $3 \mathrm{C}$-SiC, the $\mathrm{C}_{\mathrm{x}}$ atom at the saddle point of $k h, k k$, and $h h$ jumps is overcoordinated.
A more careful inspection of Figs. 9(b) and 9(c) allows us to conclude that, for the saddle-point structures of $k h$ and $k k$ jumps, besides $\mathrm{C}_{\mathrm{x}}$ having identical $\mathrm{Si}$ first neighbors, the whole $\mathrm{C}_{\mathrm{x}}$ shell is similar to that found in $3 \mathrm{C}-\mathrm{SiC}$. This means that in all three cases, $\mathrm{C}_{\mathrm{x}}$ has similar second neighbors along the MEP, at least up to the saddle point, therefore explaining their almost identical barriers of $\sim 4 \mathrm{eV}$.

Figure 9(d) shows the atomistic structure of $V_{C}$ in $4 \mathrm{H}-$ $\mathrm{SiC}$ near the saddle point along the $h h$ jump. Although the coordination of $\mathrm{C}_{\mathrm{x}}$ is similar to that found at $k h$ and $k k$ saddle points [Figs. 9(b) and 9(c), respectively], it is clear that some carbon atoms within the $\mathrm{C}_{\mathrm{x}}$ shell, namely those in the $\mathrm{Si}_{-} \mathrm{C}_{3}(k)$ structures near the arrow pointing along the $\langle 0001\rangle$ direction in Fig. 9(d), differ from the analogous $\mathrm{Si}_{-} \mathrm{C}_{3}(h)$ structures of Fig. 9(c). Importantly, for the $h h$ jump, the $\mathrm{C}_{\mathrm{x}}-\mathrm{Si}-\mathrm{C}(k)$ bond angles (with apex at $\mathrm{Si}$ ) near the transition state structure deviate less from the perfect $\mathrm{sp}^{3}$ bond angle of $109.5^{\circ}$. The $k k$ transition state shows two particularly acute $\mathrm{C}_{\mathrm{x}}-\mathrm{Si}-\mathrm{C}$ angles of $70^{\circ}$, while the lowest angle for the $h h$ transition state is $82^{\circ}$. The lower departure from perfect $\mathrm{sp}^{3}$ bonding of the $\mathrm{Si}$ atoms neighboring $\mathrm{C}_{\mathrm{x}}$ during $h h$ jumps is invoked to explain its lower activation barrier.

The investigation of migration barriers for intrinsic defects such as vacancies is crucial for understanding one of the most fundamental mechanisms of mass transport in materials, namely self-diffusion. The mechanisms for $\mathrm{Si}$ and $\mathrm{C}$ self-diffusion in SiC have previously been attributed to their corresponding intrinsic defects [68,69], and the energy barrier for $\mathrm{C}$ self-diffusion in $4 \mathrm{H}-\mathrm{SiC}$ along the $c$ axis has been experimentally estimated at $7.6 \mathrm{eV}$ [70] and $8.5 \mathrm{eV}$ [71]. However, whether $\mathrm{C}$ self-diffusion is primarily mediated by C interstitials (as proposed in Refs. [24,70] and [72]) or by vacancies (as inferred in Ref. [71]) has not been resolved experimentally. It is therefore interesting to verify the compliance of our results within the above views.

One can estimate the activation energy for self-diffusion (a process which occurs only at very high temperatures, where intrinsic conditions apply) as the sum of the formation energy and the migration barrier of the mediating defect. Under C-rich conditions (which are usually imposed during $\mathrm{SiC}$ growth to reduce the concentration of $V_{C}$ defects) the calculated formation energy of neutral $V_{\mathrm{C}}(k)$ is $E_{\mathrm{f}}=5.0 \mathrm{eV}$. For this calculation, the carbon chemical potential was considered as the energy per atom in bulk diamond (within hybrid-DFT). Hence, we anticipate activation energies for basal and axial $V_{\mathrm{C}}$-mediated carbon self-diffusion of $8.7 \mathrm{eV}$ and $9.2 \mathrm{eV}$, respectively. The latter figure is the one to be compared to the experiments referred above, where C-rich conditions are also expected, overestimating the observations by $1.6 \mathrm{eV}$ (Ref. [70]) and $0.7 \mathrm{eV}$ (Ref. [71]).

Although a detailed account of interstitial carbon $\left(\mathrm{C}_{\mathrm{i}}\right)$ in $\mathrm{SiC}$ is beyond the scope of the present work, for the sake of completeness we leave the reader with a brief analysis of carbon self-diffusion in $4 \mathrm{H}-\mathrm{SiC}$, eventually mediated by $\mathrm{C}_{\mathrm{i}}$ defects. The ground state of neutral $\mathrm{C}_{\mathrm{i}}$ has spin-1 and consists of a C-C dimer replacing a $\mathrm{C}(k)$ site [73]. In 3C-SiC the structure consists on a $\langle 001\rangle$-aligned split interstitial [73]. In C-rich material, the formation energy of a neutral $C_{i}$ defect (which is the stable state under intrinsic conditions) is $E_{\mathrm{f}}=$ $6.6 \mathrm{eV}$ (using HSE06). According to local density functional 
calculations, the estimated migration barrier of neutral $\mathrm{C}_{\mathrm{i}}$ in $3 \mathrm{C}-\mathrm{SiC}$ is estimated at $E_{\mathrm{A}}=0.5-0.6 \mathrm{eV}$ [24,72], suggesting that it could migrate already at room temperature. We note however, that the above figure is probably underestimated. If we consider that analogous calculations for the $V_{\mathrm{C}}$ migration yielded a barrier $\sim 0.5 \mathrm{eV}$ below the hybrid-DFT value [24], we arrive at $E_{\mathrm{A}} \sim 1 \mathrm{eV}$ for the estimated migration barrier of $\mathrm{C}_{\mathrm{i}}$, matching the estimated barrier for $\mathrm{C}_{\mathrm{i}}$ migration in Ref. [11]. Hence, combining this figure with the formation energy, we arrive at $\sim 7.6 \mathrm{eV}$ for the activation energy for $\mathrm{C}_{\mathrm{i}}$-mediated carbon self-diffusion in $4 \mathrm{H}-\mathrm{SiC}$. This matches the activation enthalpy measured for self-diffusion reported by Rüschenschmidt et al. [70], and it is substantially lower than the analogous quantity for $V_{\mathrm{C}}$-mediated self-diffusion, supporting a model where carbon self-diffusion in $4 \mathrm{H}-\mathrm{SiC}$ is not promoted by vacancies but rather probably by carbon interstitials $[24,70,72]$.

\section{CONCLUDING REMARKS}

We presented a detailed investigation of the mechanisms behind $V_{\mathrm{C}}$ migration in $3 \mathrm{C}$ - and $4 \mathrm{H}-\mathrm{SiC}$ based on firstprinciples calculations within hybrid DFT, combined with experimental studies using heat treatments and subsequent deep level transient depth profiling. We started by reproducing previous local-DFT results for 3C-SiC (where a migration barrier of $E_{\mathrm{A}}=3.5 \mathrm{eV}$ was previously found [24]), followed by calculations with higher $\mathbf{k}$ sampling, using the dimer method and a hybrid functional. The latter method yielded $E_{\mathrm{A}}=4.0 \mathrm{eV}$. The main reasons for the previous underestimation were (i) insufficient BZ sampling and (ii) overresonance between vacancy levels and crystalline states within the narrow gap as obtained using a (semi)local treatment to the exchange-correlation potential.

We showed that in $4 \mathrm{H}-\mathrm{SiC}$, neutral $V_{\mathrm{C}}$ migrates anisotropically and faster along basal directions. $V_{\mathrm{C}}$ migration is also found to be plane selective, meaning that the defect travels substantially faster within the $h$ plane (avoiding transiting through the $k$ plane). A total of four inequivalent jumps, labeled by pairs of sublattice sites, were identified: Two of them $\left(k h^{\prime}\right.$ and $\left.k h\right)$ contribute to axial migration, while the other two ( $k k$ and $h h)$ are involved in basal migration. Whereas $k h$ and $k k$ jumps are very similar to that occurring in $3 \mathrm{C}-\mathrm{SiC}$, including their respective barrier heights, $k h^{\prime}$ and $h h$ are singled out by showing the highest and lowest barriers, namely $E_{\mathrm{A}}=4.2 \mathrm{eV}$ and $3.7 \mathrm{eV}$, and are therefore identified as critical mechanisms governing axial and basal diffusivity of $V_{\mathrm{C}}$, respectively. While $k h^{\prime}$ hinders $V_{\mathrm{C}}$ migration due to the involvement of a high-energy twofold coordinated $\mathrm{C}$ atom at the transition state, $h h$ jumps are favored against others due to a lower departure of $\mathrm{Si}-\mathrm{C}$ bond angles from those in perfect $\mathrm{sp}^{3}$ bonded structures.

Experimentally, the activation energy for $V_{\mathrm{C}}$ diffusion in n-type $4 \mathrm{H}-\mathrm{SiC}$ was determined, and from the adherence of the experimental data to Fick's second law we firmly establish the mechanism governing $V_{\mathrm{C}}$ motion as that of fundamental vacancy migration, namely vacancy-atom exchange on the $\mathrm{C}$ sublattice. The predicted anisotropy in $V_{\mathrm{C}}$ diffusion was experimentally determined, with activation energies of $E_{\mathrm{A}}=$ $(4.4 \pm 0.3) \mathrm{eV}$ and $E_{\mathrm{A}}=(3.6 \pm 0.3) \mathrm{eV}$ for $V_{\mathrm{C}}$ migration along the $c$ and $a$ directions, respectively, and corresponding prefactors of $0.54 \mathrm{~cm}^{2} / \mathrm{s}$ and $0.017 \mathrm{~cm}^{2} / \mathrm{s}$. The magnitude of the prefactors support a simple jump process as expected for fundamental vacancy migration, and the experimentally obtained activation energies provide an excellent match with theoretical predictions of $4.2 \mathrm{eV}$ for axial and $3.7 \mathrm{eV}$ for basal $h$ sublattice $V_{\mathrm{C}}$ migration.

Finally, we examined our results within the current view of self-diffusion and whether carbon self-diffusion in $4 \mathrm{H}-\mathrm{SiC}$ is mediated by vacancies or interstitials. Although the formation energy of $V_{\mathrm{C}}$ is about $1.5 \mathrm{eV}$ lower than that of $\mathrm{C}_{\mathrm{i}}$, we support the argument that the large migration barrier of $V_{\mathrm{C}}$ makes $\mathrm{C}_{\mathrm{i}}$ the most likely mediator for carbon self-diffusion in $4 \mathrm{H}-\mathrm{SiC}$ $[24,70,72]$.

\section{ACKNOWLEDGMENTS}

Financial support was kindly provided by the Research Council of Norway and the University of Oslo through the frontier research project FUNDAMeNT (No. 251131, FriPro ToppForsk-program), and the Norwegian Micro- and Nanofabrication Facility (NorFAB 245963). Some of the computations were performed on resources provided by UNINETT Sigma2 - the National Infrastructure for High Performance Computing and Data Storage in Norway. J.C. thanks the Fundação para a Ciência e a Tecnologia (FCT) for support under project UID/CTM/50025/2019, co-funded by FEDER funds through the COMPETE 2020 Program. J.C. also acknowledges support by the NATO SPS programme (Project No. 985215). J.U.H. would like to acknowledge support from the Swedish Energy Agency Energimyndigheten project number 43611-1 and the Swedish Government Strategic Research Area in Materials Science (AFM). Some computational resources were provided by the Swedish National Infrastructure for Computing (SNIC) at HPC2N and PDC.
[1] W. Choyke and G. Pensl, MRS Bull. 22, 25 (1997).

[2] W. Bolse, Nucl. Instrum. Methods Phys. Res., Sect. B 148, 83 (1999).

[3] M. Bhatnagar and B. Baliga, IEEE Trans. Electron Devices 40, 645 (1993).
[4] T. R. Garcia, A. Kumar, B. Reinke, T. E. Blue, and W. Windl, Appl. Phys. Lett. 103, 152108 (2013).

[5] J. R. Weber, W. F. Koehl, J. B. Varley, A. Janotti, B. B. Buckley, C. G. V. de Walle, and D. D. Awschalom, Proc. Natl. Acad. Sci. 107, 8513 (2010). 
[6] L. Gordon, A. Janotti, and C. G. Van de Walle, Phys. Rev. B 92, 045208 (2015).

[7] S. Castelletto, L. Rosa, and B. C. Johnson, in Advanced Silicon Carbide Devices and Processing (InTech, Rijeka, 2015).

[8] M. Bockstedte, F. Schütz, T. Garratt, V. Ivády, and A. Gali, npj Quantum Materials 3, 31 (2018).

[9] P. B. Klein, B. V. Shanabrook, S. W. Huh, A. Y. Polyakov, M. Skowronski, J. J. Sumakeris, and M. J. O’Loughlin, Appl. Phys. Lett. 88, 052110 (2006).

[10] K. Danno, D. Nakamura, and T. Kimoto, Appl. Phys. Lett. 90, 202109 (2007).

[11] N. Iwamoto and B. G. Svensson, in Defects in Semiconductors, edited by L. Romano, V. Privitera, and C. Jagadish (Elsevier, SanDiego, 2015), Vol. 91 of Semiconductors and Semimetals, pp. 369-407.

[12] B. Zippelius, J. Suda, and T. Kimoto, J. Appl. Phys. 111, 033515 (2012).

[13] H. M. Ayedh, A. Hallén, and B. G. Svensson, J. Appl. Phys. 118, 175701 (2015).

[14] J. Coutinho, V. J. B. Torres, K. Demmouche, and S. Öberg, Phys. Rev. B 96, 174105 (2017).

[15] N. T. Son, X. T. Trinh, L. S. Løvlie, B. G. Svensson, K. Kawahara, J. Suda, T. Kimoto, T. Umeda, J. Isoya, T. Makino et al., Phys. Rev. Lett. 109, 187603 (2012).

[16] K.-B. Park, Y. Ding, J. P. Pelz, P. G. Neudeck, and A. J. Trunek, Appl. Phys. Lett. 89, 042103 (2006).

[17] J. M. Langer and H. Heinrich, Phys. Rev. Lett. 55, 1414 (1985).

[18] S. Sasaki, K. Kawahara, G. Feng, G. Alfieri, and T. Kimoto, J. Appl. Phys. 109, 013705 (2011).

[19] T. Hornos, A. Gali, and B. G. Svensson, Mater. Sci. Forum 679680, 261 (2011).

[20] H. M. Ayedh, V. Bobal, R. Nipoti, A. Hallén, and B. G. Svensson, J. Appl. Phys. 115, 012005 (2014).

[21] A. Kawasuso, F. Redmann, R. Krause-Rehberg, M. Weidner, T. Frank, G. Pensl, P. Sperr, W. Triftshäuser, and H. Itoh, Appl. Phys. Lett. 79, 3950 (2001).

[22] T. Umeda, J. Isoya, N. Morishita, T. Ohshima, and T. Kamiya, Phys. Rev. B 69, 121201(R) (2004).

[23] Z. Zolnai, N. T. Son, C. Hallin, and E. Janzén, J. Appl. Phys. 96, 2406 (2004).

[24] M. Bockstedte, A. Mattausch, and O. Pankratov, Phys. Rev. B 68, 205201 (2003).

[25] M. Bockstedte, A. Mattausch, and O. Pankratov, Phys. Rev. B 69, 235202 (2004).

[26] E. Rauls, T. Frauenheim, A. Gali, and P. Deák, Phys. Rev. B 68, 155208 (2003).

[27] F. Gao, W. J. Weber, M. Posselt, and V. Belko, Phys. Rev. B 69, 245205 (2004).

[28] X. Wang, M. Zhao, H. Bu, H. Zhang, X. He, and A. Wang, J. Appl. Phys. 114, 194305 (2013).

[29] R. Kuate Defo, X. Zhang, D. Bracher, G. Kim, E. Hu, and E. Kaxiras, Phys. Rev. B 98, 104103 (2018).

[30] L. S. Løvlie, L. Vines, and B. G. Svensson, J. Appl. Phys. 111, 103719 (2012).

[31] M. E. Bathen, H. M. Ayedh, L. Vines, I. Farkas, E. Janzén, and B. G. Svensson, Mater. Sci. Forum 924, 200 (2018).

[32] G. Kresse and J. Hafner, Phys. Rev. B 47, 558 (1993).

[33] G. Kresse and J. Hafner, Phys. Rev. B 49, 14251 (1994).

[34] G. Kresse and J. Furthmüller, Comput. Mater. Sci. 6, 15 (1996).
[35] G. Kresse and J. Furthmüller, Phys. Rev. B 54, 11169 (1996).

[36] P. E. Blöchl, Phys. Rev. B 50, 17953 (1994).

[37] J. Heyd, G. E. Scuseria, and M. Ernzerhof, J. Chem. Phys. 118, 8207 (2003).

[38] A. V. Krukau, O. A. Vydrov, A. F. Izmaylov, and G. E. Scuseria, J. Chem. Phys. 125, 224106 (2006).

[39] Z. Li and R. C. Bradt, J. Appl. Phys. 60, 612 (1986).

[40] J. Kräußlich, A. J. Bauer, B. Wunderlich, and K. Goetz, Mater. Sci. Forum 353-356, 319 (2001).

[41] J. P. Perdew, K. Burke, and M. Ernzerhof, Phys. Rev. Lett. 77, 3865 (1996).

[42] X. T. Trinh, K. Szász, T. Hornos, K. Kawahara, J. Suda, T. Kimoto, A. Gali, E. Janzén, and N. T. Son, Phys. Rev. B 88, 235209 (2013).

[43] G. Mills and H. Jónsson, Phys. Rev. Lett. 72, 1124 (1994).

[44] G. Mills, H. Jónsson, and G. K. Schenter, Surf. Sci. 324, 305 (1995).

[45] G. Henkelman and H. Jónsson, J. Chem. Phys. 111, 7010 (1999).

[46] A. Heyden, A. T. Bell, and F. J. Keil, J. Chem. Phys. 123, 224101 (2005).

[47] P. Grivickas, V. Grivickas, J. Linnros, and A. Galeckas, J. Appl. Phys. 101, 123521 (2007).

[48] J. D. Gouveia and J. Coutinho, Electron. Struct. 1, 015008 (2019).

[49] I. D. Booker, I. Farkas, I. G. Ivanov, J. Hassan, and E. Janzén, Physica. B, Condens. Matter 480, 23 (2016).

[50] J. F. Ziegler, M. Ziegler, and J. Biersack, Nucl. Instrum. Methods Phys. Res., Sect. B 268, 1818 (2010).

[51] G. Alfieri, E. V. Monakhov, B. G. Svensson, and M. K. Linnarsson, J. Appl. Phys. 98, 043518 (2005).

[52] B. G. Svensson, K.-H. Rydén, and B. M. S. Lewerentz, J. Appl. Phys. 66, 1699 (1989).

[53] P. Blood and J. W. Orton, The Electrical characterization of Semiconductors: Majority Carriers and Electron States (Academic Press Limited, London, 1992).

[54] S. D. Brotherton, Solid-State Electron. 26, 987 (1983).

[55] B. G. Svensson, C. Jagadish, A. Hallén, and J. Lalita, Phys. Rev. B 55, 10498 (1997).

[56] E. V. Monakhov, J. Wong-Leung, A. Y. Kuznetsov, C. Jagadish, and B. G. Svensson, Phys. Rev. B 65, 245201 (2002).

[57] L. Torpo, M. Marlo, T. E. M. Staab, and R. M. Nieminen, J. Phys.: Condens. Matter 13, 6203 (2001).

[58] A. Zywietz, J. Furthmüller, and F. Bechstedt, Phys. Rev. B 59, 15166 (1999).

[59] W. Kohn and L. J. Sham, Phys. Rev. 140, A1133 (1965).

[60] J. P. Perdew and A. Zunger, Phys. Rev. B 23, 5048 (1981).

[61] M. L. David, G. Alfieri, E. M. Monakhov, A. Hallén, C. Blanchard, B. G. Svensson, and J. F. Barbot, J. Appl. Phys. 95, 4728 (2004).

[62] I. Capan, T. Brodar, J. Coutinho, T. Ohshima, V. P. Markevich, and A. R. Peaker, J. Appl. Phys. 124, 245701 (2018).

[63] A. Kawasuso, H. Itoh, S. Okada, and H. Okumura, J. Appl. Phys. 80, 5639 (1996).

[64] J. W. Steeds, W. Sullivan, A. Wotherspoon, and J. M. Hayes, J. Phys.: Condens. Matter 21, 364219 (2009).

[65] T. Umeda, N. T. Son, J. Isoya, E. Janzén, T. Ohshima, N. Morishita, H. Itoh, A. Gali, and M. Bockstedte, Phys. Rev. Lett. 96, 145501 (2006). 
[66] J. Philibert, Atom movements: Diffusion and mass transport in solids, Monographies de physique (Editions de Physique, Les Ulis, 1991).

[67] H. M. Ayedh, R. Nipoti, A. Hallén, and B. G. Svensson, Appl. Phys. Lett. 107, 252102 (2015).

[68] J. Hong, M. Hon, and R. Davis, Ceramurgia Int. 5, 155 (1979).

[69] J. D. Hong and R. F. Davis, J. Am. Ceram. Soc. 63, 546 (1980).
[70] K. Rüschenschmidt, H. Bracht, N. A. Stolwijk, M. Laube, G. Pensl, and G. R. Brandes, J. Appl. Phys. 96, 1458 (2004).

[71] M. K. Linnarsson, M. S. Janson, J. Zhang, E. Janzén, and B. G. Svensson, J. Appl. Phys. 95, 8469 (2004).

[72] O. N. Bedoya-Martínez and G. Roma, Phys. Rev. B 82, 134115 (2010).

[73] A. Gali, P. Deák, P. Ordejón, N. T. Son, E. Janzén, and W. J. Choyke, Phys. Rev. B 68, 125201 (2003). 